Research Article

\title{
Performance Analysis of Conventional Beamforming Algorithm for Angle-of-Arrival Estimation under Measurement Uncertainty
}

\author{
Eun-Kyung Lee and Joon-Ho Lee \\ Department of Information and Communication Engineering, Sejong University, 209 Neungdong-ro Gwangjin-gu, Seoul 05006, \\ Republic of Korea \\ Correspondence should be addressed to Joon-Ho Lee; joonhlee@sejong.ac.kr
}

Received 11 February 2020; Revised 24 July 2020; Accepted 10 August 2020; Published 2 December 2020

Academic Editor: Rodolfo Araneo

Copyright (C) 2020 Eun-Kyung Lee and Joon-Ho Lee. This is an open access article distributed under the Creative Commons Attribution License, which permits unrestricted use, distribution, and reproduction in any medium, provided the original work is properly cited.

\begin{abstract}
The performance of the conventional beamforming for angle-of-arrival (AOA) estimation algorithm under measurement uncertainty is analyzed. Gaussian random variables are used for modeling measurement noises. Analytic expression of the mean square error (MSE) is obtained via Taylor series expansion. In traditional performance analysis, estimation accuracy in terms of the MSEs is usually obtained from the Monte Carlo simulation, which is computationally intensive especially for large number of repetitions in the Monte Carlo simulation. For reliable MSE in the Monte Carlo simulation, the number of repetitions should be very large. To circumvent this problem, analytic performance analysis which is less computationally intensive than the Monte Carlo simulation-based performance analysis is proposed in this paper. After some approximations, we derive the closed form expression of the mean square error (MSE) for each incident signal. The validity of the derived expressions is shown by comparing an analytic MSE with an empirical MSEs. The Cramer-Rao bound is also used to further validate the derived analytic expression.
\end{abstract}

\section{Introduction}

1.1. Preliminary. Determination of the angle-of-arrival (AOA) $[1,2]$ of signal has been of interest to the signal processing community. The application of the study ranges from military to civilian applications. Beamforming algorithm [3, 4], MUSIC (Multiple Signal Classification) algorithm [5], ESPRIT (Estimation of Signal Parameters via Rotational Invariance Techniques) algorithm [6], and ML (Maximum Likelihood) algorithm [7] have been the main algorithms for AOA estimation.

Beamforming algorithm is the basic AOA algorithm, whose merit is the low computational cost [2-4]. In beamforming algorithm, signals from certain directions are added constructively by forming a weighted sum of the array outputs. The antenna is steered to different directions by varying the array weights.
In this paper, performance analysis of conventional beamforming algorithm for direction-of-arrival (DOA) estimation is considered. Many studies have been conducted on conventional beamforming-based DOA estimation algorithm [8-19].

We address quantitative study on how much estimation error occurs due to an additive noise on each array antenna element. Explicit expression of mean-squared error (MSE) in terms of a standard deviation of an additive noise will be derived. In this paper, performance analysis of azimuth estimation using uniform linear array (ULA) is presented.

The estimate with no superscript and the estimate with the superscript $(u=1)$ differ due to the first approximation since the first approximation is applied in getting the estimate with the superscript $(u=1)$. Note that no approximation is applied in getting the estimate with no superscript. 
Similarly, why the estimate with the superscript $(u=1)$ and the estimate with the superscript $(u=1, v)$ differ is due to the second approximation since the first approximation and the second approximations are applied in getting the estimate with the superscript $(u=1, v)$.

Based on these observations, by comparing these three estimates, we can easily determine which approximation results in the dominant approximation error. To the best of our knowledge, no previous performance analyses have shown this intuition and no previous study presented these explicit expressions of the azimuth estimation error and the MSE of the azimuth estimate in terms of the statistics of an additive noise.

In this paper, Gaussian noise is used to model measurement uncertainty. The effect of Gaussian noise on the accuracy of the azimuth estimate is rigorously derived for DOA estimation. Furthermore, a closed-form expression of the MSE of the estimate is derived. In comparison with the previous studies on the performance analysis of the conventional beamforming algorithm, a more explicit representation of the MSE of the estimate is proposed in this paper.

Many previous studies on the conventional beamforming algorithm focused on how the performance of the conventional beamforming algorithm can be improved by proposing new algorithms or by modifying the ML DOA estimation algorithm. Note that our contribution in this paper does not lie in how much improvement can be achieved by proposing an improved beamforming DOA algorithm. Our contribution is on computational cost reduction in getting the MSE of an existing conventional beamforming algorithm by adopting an analytic approach, rather than the Monte Carlo simulation-based MSE under measurement uncertainty which can be modeled as Gaussian distributed. That is, the proposed scheme describes how analytic MSE can be obtained with much less computational complexity than the Monte Carlo simulationbased MSE.

The proposed scheme can be used in predicting how accurate the estimate of the conventional beamforming algorithm is without computationally intensive Monte Carlo simulation. The performance of the conventional beamforming algorithm depends on various parameters including the number of snapshots, the number antenna elements in the array, interelement spacing between adjacent antenna elements, and the SNR. Therefore, Monte Carlo simulations for different values of the various parameters can be computationally intensive. Therefore, the scheme presented in this paper can be adopted to predict the accuracy of the conventional beamforming-based DOA estimation algorithm for different values of various parameters.

In Table 1, the mathematical and statistical theories used for the performance analysis are illustrated. In Table 1, advantages of the proposed scheme have been described. More specifically, in Table 1, the advantage of the proposed analytical performance analysis over the Monte Carlo simulation-based empirical performance analysis is described. In addition, the advantage of the proposed analytical performance analysis over the existing analytical performance analysis method is described.

Our contribution in this manuscript does not lie in how much improvement can be achieved by proposing a new algorithm. Our contribution in this paper is to show that the MSE can be analytically derived rather than empirically. In analytic performance analysis, statistics of an additive noise, rather than noise itself, has been exploited to get the MSE of AOA estimates. On the contrary, in Monte Carlo simulation, difference noises for each repetition results in different AOA estimates, which implies that noise itself, rather than statistics of noises, is used in the Monte Carlo simulation.

In this paper, for convenience sake, measurement noises are modeled as Gaussian random variables. However, it is quite straightforward to extend to the case where the noises are modeled as different random variables as long as the moments of the random variables are available.

\section{Derivation of Analytic Expressions of Estimation Error and Mean Square Error}

2.1. Conventional Beamforming Algorithm. In conventional beamforming algorithm for AOA estimation, the array output power is computed as the arrival angle varies, and the angles corresponding to the maximum values in the output power distribution are considered to be the true directions of arrival. AOAs are selected from the angles at which the following output achieves the maximum:

$$
F(\theta)=a^{H}(\theta) \hat{R} a(\theta)=\sum_{l=1}^{M} \sum_{k=1}^{M} a_{k}^{*}(\theta) \hat{R}_{k l} a_{l}(\theta)=\sum_{l=1}^{M} \sum_{k=1}^{M} \exp \left(j\left(\psi_{l}(\theta)-\psi_{k}(\theta)\right)\right) \hat{R}_{k l}=\sum_{l=1}^{M} \sum_{k=1}^{M} P_{k l}(\theta) \hat{R}_{k l},
$$

where $\theta$ is the arrival angle of an interest and $\hat{\mathbf{R}}$ is the ML (Maximum Likelihood) estimate of the array output covariance matrix $\mathbf{R}$. That is, we evaluate (1) as a function of the arrived angle at discrete values, and find $\theta$ at which $F(\theta)$ is the maximized.

We consider the performance analysis of the conventional beamforming algorithm for multiple incident signals in this paper. In the absence of noise, the incident signal on the array antenna elements can be written as, for $i=1, \ldots, L$,

$$
x\left(t_{i}\right)=\left[\begin{array}{cccc}
a_{1}\left(\theta_{1}\right) & a_{1}\left(\theta_{2}\right) & \cdots & a_{1}\left(\theta_{N}\right) \\
a_{2}\left(\theta_{1}\right) & a_{2}\left(\theta_{2}\right) & \cdots & a_{2}\left(\theta_{N}\right) \\
\vdots & \vdots & \ddots & \vdots \\
a_{M}\left(\theta_{1}\right) & a_{M}\left(\theta_{2}\right) & \cdots & a_{M}\left(\theta_{N}\right)
\end{array}\right]\left[\begin{array}{c}
s_{1}\left(t_{i}\right) \\
s_{2}\left(t_{i}\right) \\
\vdots \\
s_{N}\left(t_{i}\right)
\end{array}\right],
$$

where $L$ is the number of snapshots. Equation (2) can be compactly written in the matrix and vector form as 
TABLe 1: Mathematical foundation and advantage of the proposed scheme.

Mathematical foundation of the proposed scheme

Empirical performance analysis

Proposed analytical performance analysis
Statistical distribution of various kinds of perturbations Higher order Taylor series expansion

Repetitive execution based on different realization of perturbation of each parameter New Monte Carlo simulation for each statistical distribtion of perturbation

Based on the statistics of perturbation rather than realizations of perturbation

Easy to accommodate various distributions of perturbations via known statistics of the distributions

$$
\mathbf{x}\left(t_{i}\right)=\mathbf{a}\left(\theta_{1}\right) s_{1}\left(t_{i}\right)+\mathbf{a}\left(\theta_{2}\right) s_{2}\left(t_{i}\right)+\cdots+\mathbf{a}\left(\theta_{N}\right) s_{N}\left(t_{i}\right) .
$$

The noisy array response, denoted by $\mathbf{x}^{\prime}\left(t_{i}\right)$, can be expressed as

$$
x^{\prime}\left(t_{i}\right)=\left[\begin{array}{cccc}
a_{1}\left(\theta_{1}\right) & a_{1}\left(\theta_{2}\right) & \cdots & a_{1}\left(\theta_{N}\right) \\
a_{2}\left(\theta_{1}\right) & a_{2}\left(\theta_{2}\right) & \cdots & a_{2}\left(\theta_{N}\right) \\
\vdots & \vdots & \ddots & \vdots \\
a_{M}\left(\theta_{1}\right) & a_{M}\left(\theta_{2}\right) & \cdots & a_{M}\left(\theta_{N}\right)
\end{array}\right]\left[\begin{array}{c}
s_{1}\left(t_{i}\right) \\
s_{2}\left(t_{i}\right) \\
\vdots \\
s_{N}\left(t_{i}\right)
\end{array}\right]+\left[\begin{array}{c}
n_{1}\left(t_{i}\right) \\
n_{2}\left(t_{i}\right) \\
\vdots \\
n_{M}\left(t_{i}\right)
\end{array}\right] .
$$

In matrix and vector form,(4) can be expressed as

$$
\mathbf{x}^{\prime}\left(t_{i}\right)=\mathbf{a}\left(\theta_{1}\right) s_{1}\left(t_{i}\right)+\mathbf{a}\left(\theta_{2}\right) s_{2}\left(t_{i}\right)+\cdots+\mathbf{a}\left(\theta_{N}\right) s_{N}\left(t_{i}\right)+\mathbf{n}\left(t_{i}\right) .
$$

Noiseless array responses due to the $n$th incident signal, $s_{n}(t)$, are denoted by $\mathbf{x}^{(n)}(t)$ :

$$
\mathbf{x}^{(n)}\left(t_{i}\right) \equiv \mathbf{a}\left(\theta_{n}\right) s_{n}\left(t_{i}\right) .
$$

The sample covariance matrices associated with $\mathbf{x}\left(t_{i}\right)$ and $\mathbf{x}^{\prime}\left(t_{i}\right)$ are defined as

$$
\begin{aligned}
\hat{\mathbf{R}} & =\frac{1}{L} \sum_{i=1}^{L} \mathbf{x}\left(t_{i}\right) \mathbf{x}^{H}\left(t_{i}\right), \\
\hat{\mathbf{R}}^{\prime} & =\frac{1}{L} \sum_{i=1}^{L} \mathbf{x}^{\prime}\left(t_{i}\right) \mathbf{x}^{{ }^{H}}\left(t_{i}\right) .
\end{aligned}
$$

Note that, in (7) and (8), all the incident signals are taken into account.

Let $\hat{\mathbf{R}}^{(n)}$ denotes the sample covariance matrices associated with the $n$th incident signal for noiseless case. Similarly, $\hat{\mathbf{R}}^{\prime(n)}$ denotes the sample covariance matrix associated with the $n$th incident signal for noisy case. Sample covariance matrix associated with the $n$th incident signal can be expressed as

$$
\hat{\mathbf{R}}^{(n)}=\frac{1}{L} \sum_{i=1}^{L} \mathbf{x}^{(n)}\left(t_{i}\right)\left(\mathbf{x}^{(n)}\left(t_{i}\right)\right)^{H}
$$

From (9), it is can be easily shown that the entries of $\hat{\mathbf{R}}^{(n)}$ can be expressed as

$$
\hat{R}_{k l}^{(n)}=\frac{1}{L} \sum_{i=1}^{L} x_{k}^{(n)}\left(t_{i}\right)\left(x_{l}^{(n)}\left(t_{i}\right)\right)^{*} .
$$

The difference between $\hat{\mathbf{R}}^{\prime}$ in (8) and $\hat{\mathbf{R}}^{(n)}$ in (9) is defined as $\delta \hat{\mathbf{R}}$ :

$$
\delta \hat{\mathbf{R}}^{(n)}=\hat{\mathbf{R}}^{\prime}-\hat{\mathbf{R}}^{(n)} .
$$

Let $\theta_{n}^{(0)}$ denote the true azimuth angle of the $n$th incident signal. The entries of $\delta \hat{\mathbf{R}}^{(n)}$ can be expressed as

$$
\delta \hat{R}_{k l}^{(n)}=\frac{1}{L} \sum_{i=1}^{L}\left(x_{k}^{(n)}\left(t_{i}\right)\left(n_{l}^{(n)}\left(t_{i}\right)\right)^{*}+n_{k}^{(1)}\left(t_{i}\right)\left(x_{l}^{(1)}\left(t_{i}\right)\right)^{*}+n_{k}\left(t_{i}\right)\left(n_{l}^{(n)}\left(t_{i}\right)\right)^{*}\right) .
$$

For noiseless case, AOA of each signal is estimated from the angle satisfying

$$
\hat{\theta}_{n}=\arg \max _{\theta} F^{(n)}(\theta),
$$

where is defined as

$$
F^{(n)}(\theta)=\mathbf{a}^{H}(\theta) \hat{\mathbf{R}}^{(n)} \mathbf{a}(\theta)=\sum_{l=1}^{M} \sum_{k=1}^{M} a_{k}^{*}(\theta) \widehat{R}_{k l}^{(n)} a_{l}(\theta)=\sum_{l=1}^{M} \sum_{k=1}^{M} \exp \left(j\left(\psi_{l}(\theta)-\psi_{k}(\theta)\right)\right) \hat{R}_{k l}^{(n)}=\sum_{l=1}^{M} \sum_{k=1}^{M} P_{k l}(\theta) \hat{R}_{k l}^{(n)}
$$


Note that $P_{k l}(\theta)$ in (14) is defined as

$$
P_{k l}(\theta)=\exp \left(j\left(\psi_{l}(\theta)-\psi_{k}(\theta)\right)\right)=\exp \left(j \frac{2 \pi}{\lambda} \Delta \sin \theta(l-k)\right) .
$$

(13) follows from the fact that the AOA is obtained from the angle maximizing $F^{(n)}(\theta)$. The angle maximizing $F^{(n)}(\theta)$ can be obtained from

$$
\frac{\mathrm{d}}{\mathrm{d} \theta} F^{(n)}(\theta)=0
$$

In the case of noisy response, $\mathrm{AOA}$ is estimated from the angle satisfying

$$
\hat{\theta}_{n}=\arg \max _{\theta} F^{\prime}(\theta),\left(\theta_{n}^{(0)}-\Delta \theta<\theta<\theta_{n}^{(0)}+\Delta \theta\right),
$$

where $F^{\prime}(\theta)$ is defined as

$$
F^{\prime}(\theta)=\mathbf{a}^{H}(\theta) \hat{\mathbf{R}}^{\prime} a(\theta)=\sum_{l=1}^{M} \sum_{k=1}^{M} a_{k}^{*}(\theta) \hat{R}_{k l}^{\prime} a_{l}(\theta)=\sum_{l=1}^{M} \sum_{k=1}^{M} \exp \left(j\left(\psi_{l}(\theta)-\psi_{k}(\theta)\right)\right) \hat{R}_{k l}^{\prime}=\sum_{l=1}^{M} \sum_{k=1}^{M} P_{k l}(\theta) \hat{R}_{k l}^{\prime}
$$

(17) follows from the fact that the AOA is obtained from the angle maximizing $F^{\prime}(\theta)$. The angle maximizing $F^{\prime}(\theta)$ can be given by

$$
\frac{\mathrm{d}}{\mathrm{d} \theta} F^{\prime}(\theta)=0
$$

2.2. Closed-Form Expression of Estimation Error. In the absence of an additive noise, the estimates of the AOAs should satisfy

$$
\begin{aligned}
\left.\frac{\mathrm{d}}{\mathrm{d} \theta} F^{(n)}(\theta)\right|_{\theta=\theta_{n}^{(0)}} & =\left.\sum_{l=1}^{M} \sum_{k=1}^{M} \frac{\mathrm{d}}{\mathrm{d} \theta} P_{k l}(\theta) \hat{R}_{k l}^{(n)}\right|_{\theta=\theta_{n}^{(0)}} \\
& =\left.\sum_{l=1}^{M} \sum_{k=1}^{M} f_{k l}(\theta) \hat{R}_{k l}^{(n)}\right|_{\theta=\theta_{n}^{(0)}}=0,
\end{aligned}
$$

where $f_{k l}(\theta)$ is defined from

$$
f_{k l}(\theta)=\frac{d}{d \theta} P_{k l}(\theta)
$$

Similarly, when there is an additive noise, the estimates of the AOAs, $\hat{\theta}_{n}$, are determined from the angle satisfying

$$
\begin{aligned}
\left.\frac{\mathrm{d}}{\mathrm{d} \theta} F^{\prime}(\theta)\right|_{\theta=\theta_{n}^{(0)}} & =\left.\sum_{l=1}^{M} \sum_{k=1}^{M} \frac{\mathrm{d}}{\mathrm{d} \theta} P_{k l}(\theta) \hat{R}_{k l}^{\prime}\right|_{\theta=\theta_{n}^{(0)}} \\
& =\left.\sum_{l=1}^{M} \sum_{k=1}^{M} f_{k l}(\theta) \hat{R}_{k l}^{\prime}\right|_{\theta=\theta_{n}^{(0)}}=0 .
\end{aligned}
$$

Using $f_{k l}(\theta)$ in (20), (22) is given by

$$
f_{k l}(\theta)=\exp \left(j \frac{2 \pi}{\lambda} \Delta \sin \theta(l-k)\right)\left(j \frac{2 \pi}{\lambda} \Delta \cos \theta(l-k)\right) .
$$

Let $\delta \theta_{n}$ represent estimation errors of the $n$th incident signal:

$$
\hat{\theta}_{n}=\theta_{n}^{(0)}+\delta \theta_{n}
$$

where $\hat{\theta}_{n}$ is an estimate of the $n$th incident signal and $\hat{\theta}_{n}^{(0)}$ is the true azimuth of the $n$th incident signal.

Substituting (24) in (20), we have

$$
\left.\frac{\mathrm{d}}{\mathrm{d} \theta} F^{(n)}(\theta)\right|_{\theta=\theta_{n}^{(0)}+\delta \theta_{n}}=\sum_{l=1}^{M} \sum_{k=1}^{M} f_{k l}\left(\theta_{n}^{(0)}+\delta \theta_{n}\right)\left(\hat{R}_{k l}^{(n)}+\delta \hat{R}_{k l}^{(n)}\right)=0
$$

We can rewrite (25) as

$$
\sum_{l=1}^{M} \sum_{k=1}^{M} f_{k l}\left(\theta_{n}^{(0)}+\delta \theta_{n}^{(u=1)}\right)\left(\hat{R}_{k l}^{(n)}+\delta \hat{R}_{k l}^{(n)}\right) \approx \sum_{l=1}^{M} \sum_{k=1}^{M}\left(f_{k l}\left(\theta_{n}^{(0)}\right)+\left.\delta \theta_{n}^{(u=1)} \frac{\partial}{\partial \theta} f_{k l}(\theta)\right|_{\theta=\theta_{n}^{(0)}}\right)\left(\hat{R}_{k l}^{(n)}+\delta \hat{R}_{k l}^{(n)}\right)=0
$$

where the superscript $(u=1)$ denotes that the first-order Taylor series expansion is adopted. We can rewrite (26) as

$$
\sum_{l=1}^{M} \sum_{k=1}^{M}\left(f_{k l}\left(\theta_{n}^{(0)}\right) \hat{R}_{k l}^{(n)}\right)+\sum_{l=1}^{M} \sum_{k=1}^{M}\left(f_{k l}\left(\theta_{n}^{(0)}\right) \delta \hat{R}_{k l}^{(n)}\right)+\delta \theta_{n}^{(u=1)} \sum_{l=1}^{M} \sum_{k=1}^{M}\left(\left.\frac{\mathrm{d}}{\mathrm{d} \theta} f_{k l}(\theta)\right|_{\theta=\theta_{n}^{(0)}} \hat{R}_{k l}^{(n)}\right)+\delta \theta_{n}^{(u=1)} \sum_{l=1}^{M} \sum_{k=1}^{M}\left(\frac{\mathrm{d}}{\mathrm{d} \theta} f_{k l}(\theta) \mid \theta=\theta_{n}^{(0)} \delta \hat{R}_{k l}^{(n)}\right)=0
$$


Note that, from (20), the first term of the right-hand side of (27) is identically zero. Therefore, (27) reduces to

$$
\delta \theta_{n}^{(u=1)} \sum_{l=1}^{M} \sum_{k=1}^{M}\left(\left.\frac{\mathrm{d}}{\mathrm{d} \theta} f_{k l}(\theta)\right|_{\theta=\theta_{n}^{(0)}}\left(\hat{R}_{k l}^{(n)}+\delta \hat{R}_{k l}^{(n)}\right)\right)=-\sum_{l=1}^{M} \sum_{k=1}^{M}\left(f_{k l}\left(\theta_{n}^{(0)}\right) \delta \hat{R}_{k l}^{(n)}\right) .
$$

From (28), $\delta \theta_{n}^{(u=1)}$ can be explicitly written as

$$
\delta \theta_{n}^{(u=1)}=\frac{-\sum_{l=1}^{M} \sum_{k=1}^{M}\left(f_{k l}\left(\theta_{n}^{(0)}\right) \delta \hat{R}_{k l}^{(n)}\right)}{\sum_{l=1}^{M} \sum_{k=1}^{M}\left(\left.(\mathrm{~d} / \mathrm{d} \theta) f_{k l}(\theta)\right|_{\theta=\theta_{n}^{(0)}}\left(\hat{R}_{k l}^{(n)}+\delta \hat{R}_{k l}^{(n)}\right)\right)} .
$$

Since the fourth term of the right-hand side (RMS) of (27) is much less than the third term of the right-hand side of (27) and (28), it can be approximated as

$\sum_{l=1}^{M} \sum_{k=1}^{M}\left(f_{k l}\left(\theta_{n}^{(0)}\right) \delta \hat{R}_{k l}^{(n)}\right)+\delta \theta_{n}^{(u=1, v)} \sum_{l=1}^{M} \sum_{k=1}^{M}\left(\left.\frac{\mathrm{d}}{\mathrm{d} \theta} f_{k l}(\theta)\right|_{\theta=\theta_{n}^{(0)}} \hat{R}_{k l}^{(n)}\right)=0$, where the superscript $v$ indicates that the fourth term of the RMS of (27) has been omitted. From (30), $\delta \theta_{n}^{(u=1, v)}$ can be evaluated as

$$
\delta \theta_{n}^{(u=1, v)}=\frac{-\sum_{l=1}^{M} \sum_{k=1}^{M}\left(f_{k l}\left(\theta_{n}^{(0)}\right) \delta \hat{R}_{k l}^{(n)}\right)}{\left(\sum_{l=1}^{M} \sum_{k=1}^{M}(\mathrm{~d} / \mathrm{d} \theta) f_{k l}(\theta) \mid \theta=\theta_{n}^{(0)} \hat{R}_{k l}^{(n)}\right)}
$$

$$
\begin{aligned}
\frac{\mathrm{d}}{\mathrm{d} \theta} f_{k l}(\theta)= & \frac{\mathrm{d}}{\mathrm{d} \theta} \exp \left(j \frac{2 \pi}{\lambda}(l-k) \Delta \sin \theta\right)\left(j \frac{2 \pi}{\lambda}(l-k) \Delta \cos \theta\right)=\exp \left(j \frac{2 \pi}{\lambda}(l-k) \Delta \sin \theta\right)\left(j \frac{2 \pi}{\lambda}(l-k) \Delta \cos \theta\right)^{2} \\
& +\exp \left(j \frac{2 \pi}{\lambda}(l-k) \Delta \sin \theta\right)\left(-j \frac{2 \pi}{\lambda}(l-k) \Delta \sin \theta\right) .
\end{aligned}
$$

2.3. Closed-Form Expression of Mean Square Error. From

(31), $E\left[\left(\delta \theta_{n}^{(u=1, v)}\right)^{2}\right]$ is given by

$$
\begin{aligned}
E\left[\left(\delta \theta_{n}^{(u=1, v)}\right)^{2}\right] & =E\left[\delta \theta_{n}^{(u=1, v)}\left(\delta \theta_{n}^{(u=1, v)}\right)^{*}\right]=E\left[\left(\frac{-\sum_{l=1}^{M} \sum_{k=1}^{M}\left(f_{k l}\left(\theta_{n}^{(0)}\right) \delta \hat{R}_{k l}^{(n)}\right)}{\sum_{l=1}^{M} \sum_{k=1}^{M}\left(\left.(\mathrm{~d} / \mathrm{d} \theta) f_{k l}(\theta)\right|_{\left.\theta=\theta_{2}^{(0)} \hat{R}_{k l}^{(n)}\right)}\right)}\right)\left(\frac{-\sum_{l=1}^{M} \sum_{k=1}^{M}\left(f_{k l}\left(\theta_{n}^{(0)}\right) \delta \hat{R}_{k l}^{(n)}\right)}{\sum_{l=1}^{M} \sum_{k=1}^{M}\left(\left.(\mathrm{~d} / \mathrm{d} \theta) f_{k l}(\theta)\right|_{\theta=\theta_{n}^{(0)}} \hat{R}_{k l}^{(n)}\right)}\right)^{*}\right] \\
& =\frac{\sum_{l=1}^{M} \sum_{k=1}^{M} \sum_{l^{\prime}=1}^{M} \sum_{k^{\prime}=1}^{M} f_{k l}\left(\theta_{n}^{(0)}\right) f_{k^{\prime} l^{\prime}}^{*}\left(\theta_{n}^{(0)}\right) E\left(\delta \hat{R}_{k l}^{(n)}\left(\delta \hat{R}_{k^{\prime} l^{\prime}}^{(n)}\right)^{*}\right)}{\left.\left.\sum_{l=1}^{M} \sum_{k=1}^{M} \sum_{l^{\prime}=1}^{M} \sum_{k^{\prime}=1}^{M}(\mathrm{~d} / \mathrm{d} \theta) f_{k l}(\theta)\right|_{\theta=\theta_{n}^{(0)}}(\mathrm{d} / \mathrm{d} \theta) f_{k^{\prime} l^{\prime}}^{*}(\theta)\right|_{\theta=\theta_{n}^{(0)}} \hat{R}_{k l}^{(n)}\left(\hat{R}_{k^{\prime} l^{\prime}}^{(n)}\right)^{*}}
\end{aligned}
$$


where the first equality holds since $\delta \theta_{n}^{(u=1, v)}$ is real valued. The entries of $E\left(\delta \hat{R}_{k l}^{(n)}\left(\delta \hat{R}_{k^{\prime} l^{\prime}}^{(n)}\right)^{*}\right)$ in (33) can be found in the A-D.

\section{Cramer-Rao Bound}

The lower bound of the MSE for an unbiased estimator is called the Cramer-Rao bound (CRB) [20]. The CRB of each parameter can be obtained from the diagonal entry of $\mathbf{B}_{\mathrm{STO}}$ :

$$
\begin{aligned}
E\left[\left\{\hat{\Theta}-\Theta^{(0)}\right\}\left\{\hat{\Theta}-\Theta^{(0)}\right\}^{T}\right] & \geq \mathbf{B}_{\text {STO }} \\
\hat{\Theta} & =\left[\begin{array}{llll}
\hat{\theta}_{1} & \hat{\theta}_{2} & \ldots & \hat{\theta}_{N}
\end{array}\right]^{T}, \\
\Theta^{(0)} & =\left[\begin{array}{llll}
\theta_{1}^{(0)} & \theta_{2}^{(0)} & \ldots & \theta_{N}^{(0)}
\end{array}\right]^{T}, \\
\left(\mathbf{B}_{\text {STO }}^{-1}\right)_{i j} & =\frac{2 N}{\sigma^{2}} \operatorname{Re}\left[\operatorname{Tr}\left\{\mathbf{A}_{j}^{H} \mathbf{P}_{\mathbf{A}}^{\perp} \mathbf{A}_{i} \mathbf{S A}^{H} \mathbf{R}^{-1} \mathbf{A S}\right\}\right], \quad i, j=1, \ldots, p N,
\end{aligned}
$$

where $N$ is the number of incident signals and $p$ is the number of parameters to be estimated for each incident signal. For estimation of azimuth, $p$ is equal to one. For estimation of azimuth and elevation, $p$ is equal to two. $\mathbf{A}$ is a $M$ by $N$ matrix. Each column of $\mathbf{A}$ is an array vector associated with each incident signal. $\mathbf{P}_{\mathbf{A}}$ is a projection matrix onto the column space of $\mathbf{A}$. $\mathbf{S}$ is source covariance matrix, and $\mathbf{R}$ is array covariance matrix.

\section{Summary}

In this section, the performance analysis scheme presented in this paper is summarized. The performance analysis scheme and mathematical details on the performance analysis are shown in Figure 1.

In Figure 2, it is clearly shown how the proposed scheme is different from the existing method in [21]. In [21], an implicit expression of estimation error is derived. Note that $\Delta \alpha_{1}$ is a transformed variable of an azimuth, not the azimuth itself. On the contrary, in this paper, explicit expressions of estimation error of the azimuth itself and the MSE of the azimuth itself have been derived, as shown in Figure 2. In addition, it can be shown that the scheme presented in this paper can be extended to the simultaneous estimation of azimuth and elevation.

\section{Numerical Results}

The parameters for simulation are as follows:

(1) Search range of azimuth $(\theta):-90^{\circ} \sim 90^{\circ}$

(2) Search step: $1^{\circ}$

(3) The number of incident signals $(N): 2$

(4) The number of antennas $(M): 10$

(5) Distance between adjacent antenna elements: $0.3 \lambda$ and $0.5 \lambda$.

The number of Monte Carlo simulation, $T$, is chosen to be 10,000. Therefore, 10,000 Monte Carlo repetitions are performed and the empirical MSEs are calculated:

$$
\begin{gathered}
E\left[\left(\hat{\theta}_{n}^{\prime}-\theta_{n}^{(0)}\right)^{2}\right]=E\left[\delta \theta_{n} \delta \theta_{n}^{*}\right]=\frac{1}{T} \sum_{t=1}^{T}\left(\left(\delta \theta_{n}\right)_{(t)}\right)^{2}, \\
E\left[\left(\hat{\theta}_{n}^{(u=1)}-\theta_{n}^{(0)}\right)^{2}\right]=E\left[\delta \theta_{n}^{(u=1)}\left(\delta \theta_{n}^{(u=1)}\right)^{*}\right]=\frac{1}{T} \sum_{t=1}^{T}\left(\left(\delta \theta_{n}^{(u=1)}\right)_{(t)}\right)^{2}, \\
E\left[\left(\hat{\theta}_{n}^{(u=1, v)}-\theta_{n}^{(0)}\right)^{2}\right]=E\left[\delta \theta_{n}^{(u=1, v)}\left(\delta \theta_{n}^{(u=1, v)}\right)^{*}\right]=\frac{1}{T} \sum_{t=1}^{T}\left(\left(\delta \theta_{n}^{(u=1, v)}\right)_{(t)}\right)^{2},
\end{gathered}
$$

where the subscript " $(t)$ " denotes the $t$ th repetition out of $T$ repetitions.

\subsection{Estimation with Sensor Array of Elemental Distance $0.3 \lambda$.} The correlation coefficient between two incident signals is set to be zero, which quantifies the correlation coefficient for uncorrelated incident signals. Widely separated signals from $\theta_{1}^{(0)}=-40^{\circ}$ and $\theta_{2}^{(0)}=65^{\circ}$ are considered.

The results for elemental distances of $0.3 \lambda$ are illustrated in Figures 3 and 4, respectively. Figure 3 illustrates how MSEs change as the number of snapshots and the SNR increase. In Figure 3, it is shown that the empirical MSEs of $\hat{\theta}_{1}^{\prime}(u=1)$ and $\hat{\theta}_{1}^{\prime(u=1, v)}$ with respect to the number of snapshots 


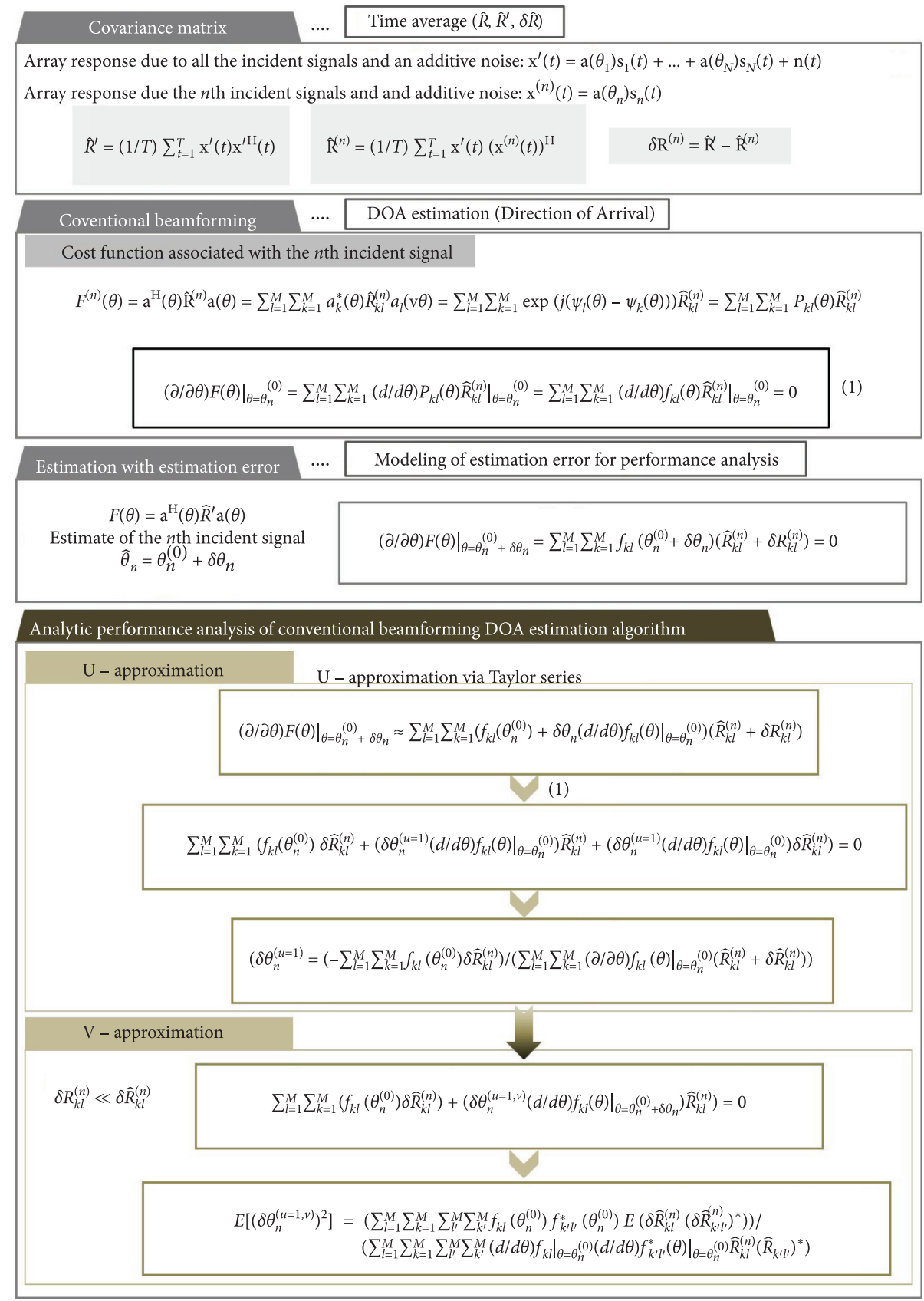

Figure 1: Performance analysis of conventional beamforming.

are close to that of $\hat{\theta}_{1}^{\prime}$. It is also shown that the analytic MSE of $\hat{\theta}_{1}^{(u=1, v)}$ is in perfect agreement with the empirical MSE of $\hat{\theta}_{1}^{\prime(u=1, v)}$. Consistency of the analytic MSE with the CRB is also illustrated in Figure 3, where $T$ is equal to 10,000 .

Figure 4 illustrates the validity of various derived expressions associated with the second incident signal by comparing the analytic result with the simulation results. It is also shown that the CRB is close to the analytic MSE and the empirical MSEs.

It is also clear that the MSEs with the superscript " $u=1$ " and the superscripts " $u=1, v$ " are quite close to that with no approximation, which illustrates that two approximations associated with superscript " $u=1, v$ " are valid. It is also shown that the MSE of analytic result and the MSE of the simulation results with superscript " $(u=1, v)$ " show good agreement. The decrease of MSEs with the increase of SNR is quite clear for all the results.

5.2. Closely Spaced Incident Signals. In this section, it is shown whether the proposed algorithm works for the closedly spaced incident signals for elemental distances of $0.5 \lambda$. The correlation coefficient between two incident signals is set to be zero. Closely separated signals from $\theta_{1}^{(0)}=60^{\circ}$ and $\theta_{2}^{(0)}=65^{\circ}$ are considered. In Figure 5, the results for elemental distances of $0.5 \lambda$ are illustrated. 


\section{Existing method}

1. Implicit expression of estimation error of transformed variable of azimuth.

$$
\begin{aligned}
& \Delta \alpha_{1}=\left[-\left(\mathrm{A}_{12} \mid c \cos \left(\measuredangle C_{12}\right) M+A_{2} g_{2}\left(\alpha_{1}\right)\right] g_{2}^{\prime}\left(\alpha_{1}\right) /\right. \\
& {\left[A_{12} M g_{2}^{\prime \prime}\left(\alpha_{1}\right)+A_{2}\left[g_{2}^{\prime 2}\left(\alpha_{1}\right)+g_{2}\left(\alpha_{1}\right) g_{2}^{\prime \prime}\left(\alpha_{1}\right)\right]+A_{12} \mid c \cos \left(\measuredangle C_{12}\right)\left[M g_{2}^{\prime \prime}\left(\alpha_{1}\right)+g_{2}\left[\left(\alpha_{1}\right)+g_{1}^{\prime \prime}\left(\alpha_{1}\right)\right]\right]\right.}
\end{aligned}
$$

2. No expression of MSE.

\section{Proposed method}

1. Explicit expression of azimuth estimation error.

$$
\left.\delta \theta_{n}{ }^{(u=1, v)}=\left(-\sum_{l=1}^{M} \sum_{k=1}^{M}\left(f_{k l}\left(\theta_{n}^{(0)}\right) \delta \hat{R}_{k l}^{(n)}\right)\right) /\left(\left.\sum_{l=1}^{M} \sum_{k=1}^{M}(\partial / \partial \theta) f_{k l}(\theta)\right|_{\theta=\theta_{n}} \delta \hat{k}_{k l}^{(n)}\right)\right), n=1, \ldots, N
$$

2. Explicit expression of MSE.

$$
\begin{aligned}
E\left[\left(\delta \theta_{n}^{(u=1, v)}\right)^{2}\right]= & \left(\sum_{l=1}^{M} \sum_{k=1}^{M} \sum_{l^{\prime}=1}^{M} \sum_{k^{\prime}=1}^{M} f_{k l}\left(\theta_{n}^{(0)}\right) f_{k^{\prime} l^{\prime}}^{*}\left(\theta_{n}^{(0)}\right) E\left(\delta \widehat{R}_{k l}^{(n)}\left(\delta \widehat{R}_{k^{\prime} l^{\prime}}\right)^{*}\right)\right) / \\
& \left.\left.\left(\sum_{l=1}^{M} \sum_{k=1}^{M} \sum_{l^{\prime}=1}^{M} \sum_{k^{\prime}=1}^{M}(\partial / \partial \theta) f_{k l}\right) \theta\right)\left.\right|_{\left.\theta=\left.\theta_{n}^{(0)}(\partial / \partial \theta) f_{k}^{*(\eta)}(\theta)\right|_{\theta=\theta_{n}} ^{(0)} \widehat{R}_{k l}^{(n)}\left(\widehat{R}_{k^{\prime}}^{(n)}\right)^{*}\right)}\right)
\end{aligned}
$$

FIgURE 2: Comparison of the proposed scheme with the existing method.

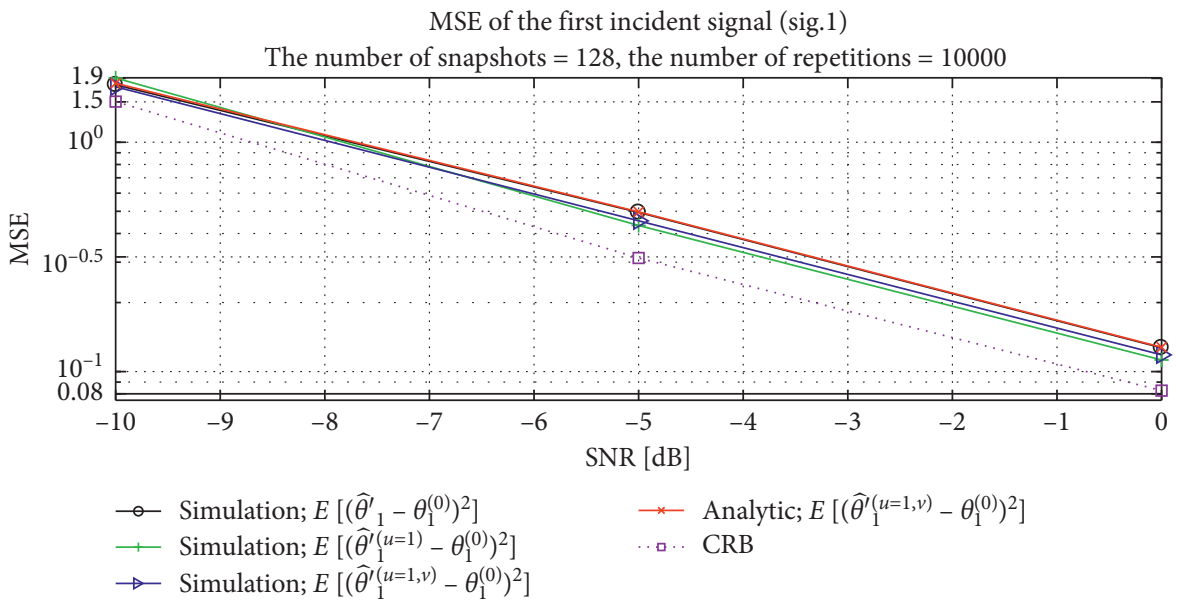

(a)

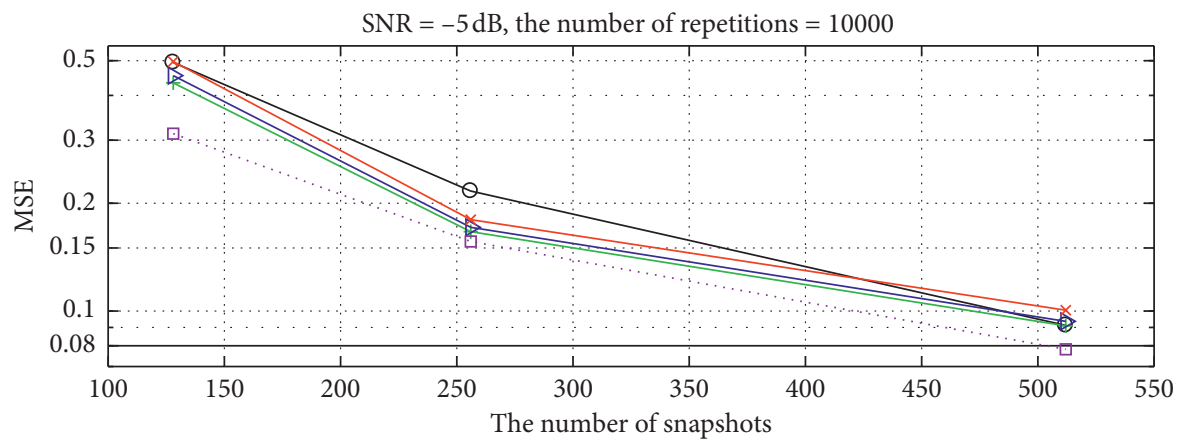

$\rightarrow$ Simulation; $E\left[\left(\widehat{\theta}^{\prime} 1-\theta_{1}^{(0)}\right)^{2}\right]$
$\rightarrow$ Simulation; $E\left[\left(\widehat{\theta}^{\prime}(1=1)-\theta_{1}^{(0)}\right)^{2}\right]$
$\rightarrow$ Simulation; $E\left[\left(\widehat{\theta}_{1}^{\prime(u=1, v)}-\theta_{1}^{(0)}\right)^{2}\right]$

$$
\begin{aligned}
& \rightarrow \text { Analytic; } E\left[\left(\hat{\theta}_{1}^{\prime(u=1, v)}-\theta_{1}^{(0)}\right)^{2}\right] \\
& \therefore \square \text { CRB }
\end{aligned}
$$

(b)

FIgURE 3: MSEs and CRB of the first incident signal. 


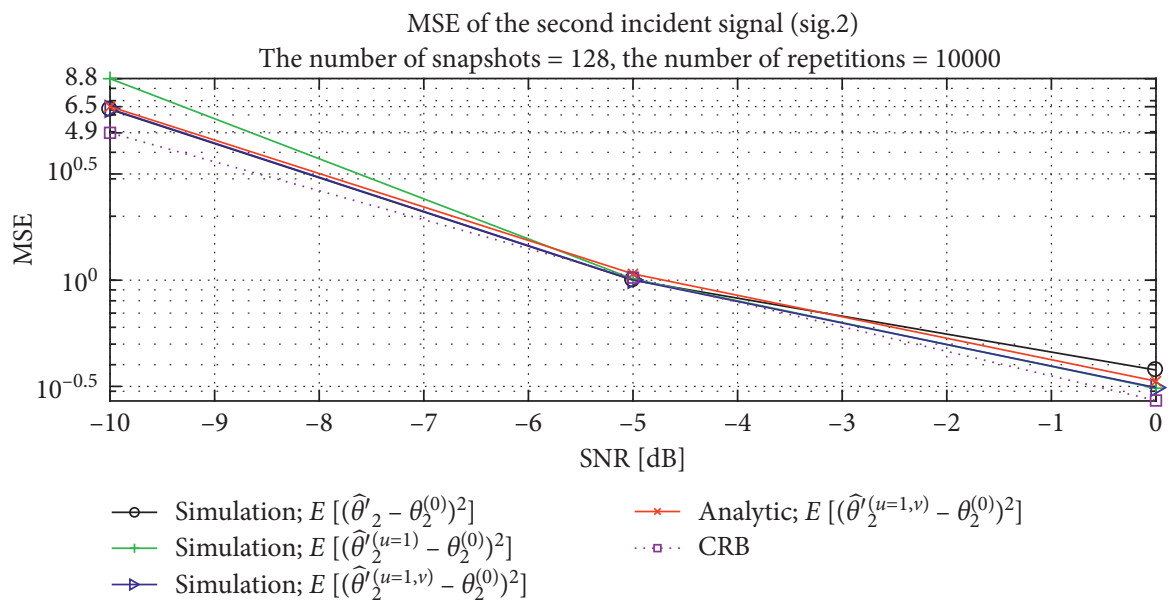

(a)

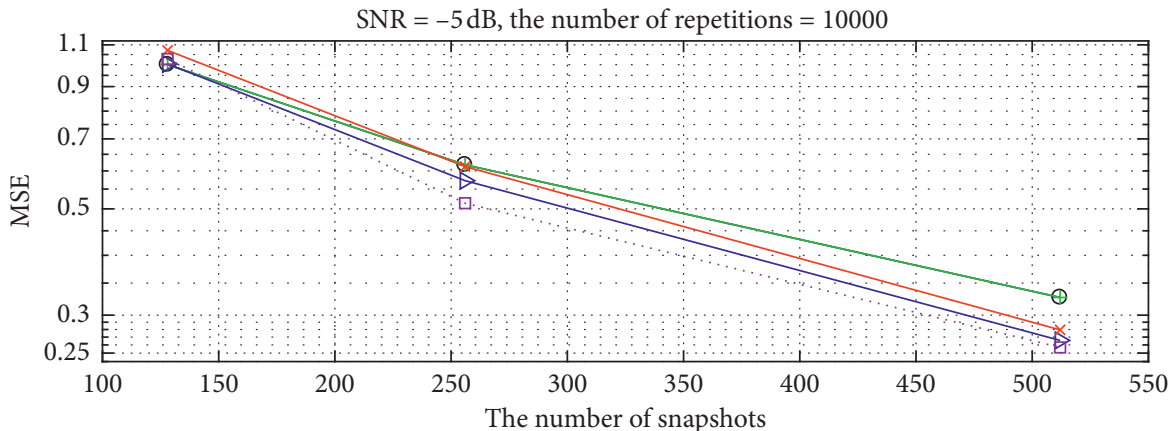

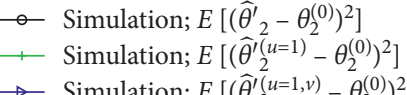

$\star \quad$ Analytic; $E\left[\left(\widehat{\theta}_{2}^{\prime(u=1, v)}-\theta_{2}^{(0)}\right)^{2}\right]$

a.. CRB

(b)

FIgURE 4: MSEs and CRB of the second incident signal.

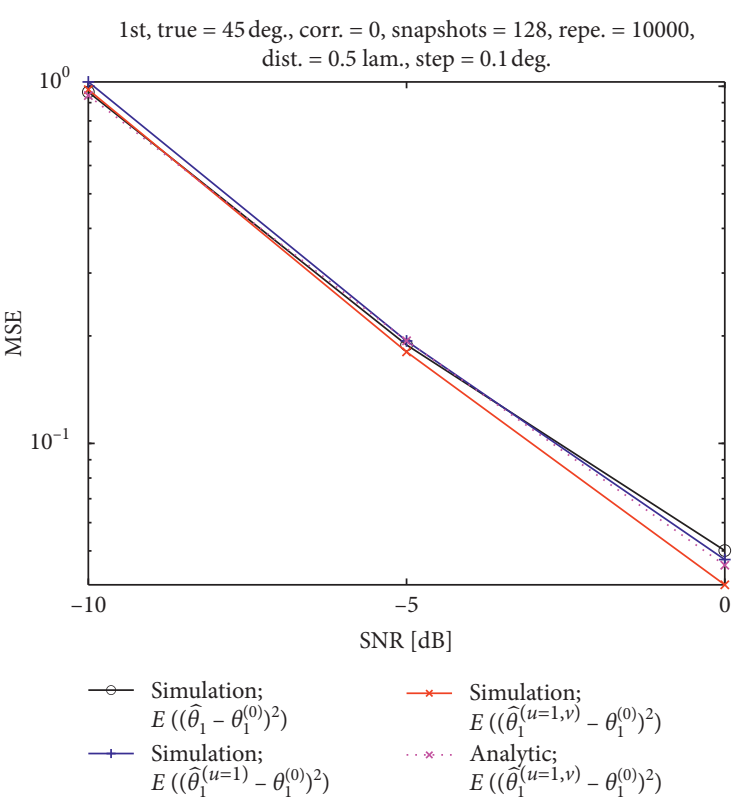

(a)

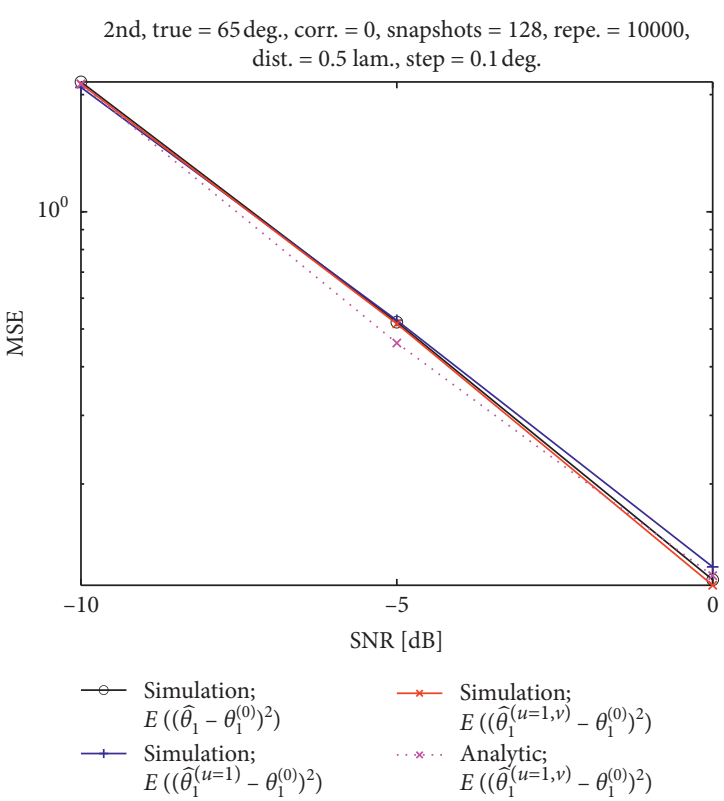

(b)

FIgURE 5: Continued. 


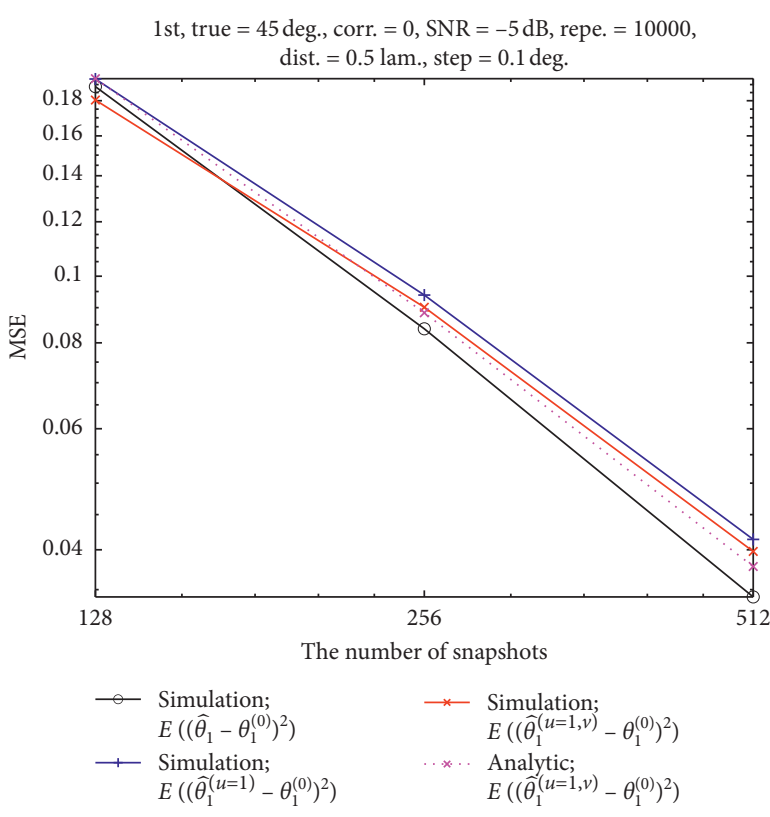

(c)

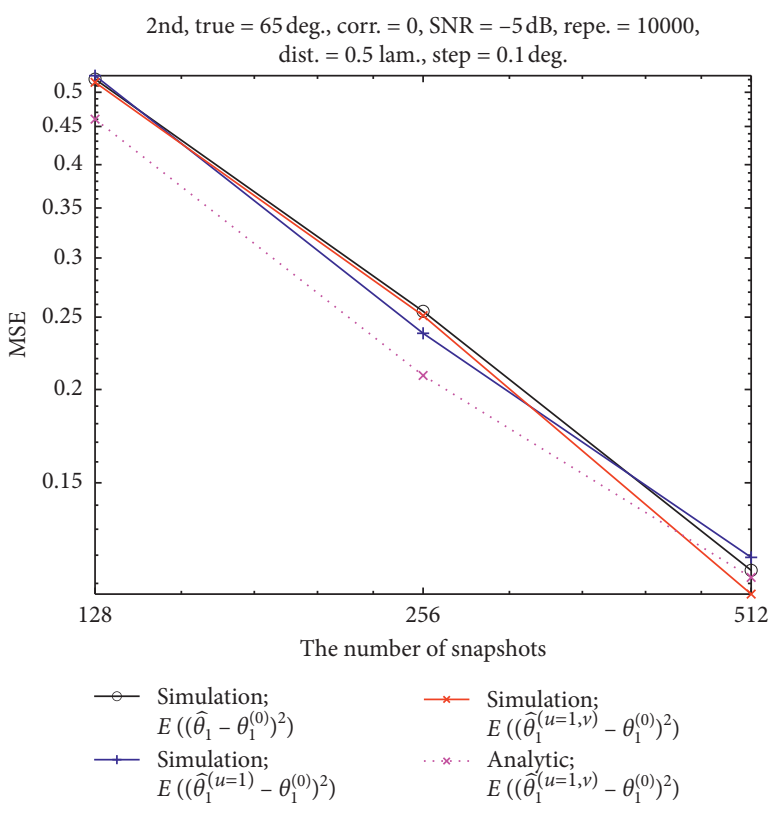

(d)

Figure 5: MSEs of analytic approach and that of Monte Carlo simulation-based approach (closely spaced signals from $\theta_{1}^{(0)}=45^{\circ}$ and $\theta_{2}^{(0)}=65^{\circ}$ ): (a) first incident signal $\left(\theta_{1}^{(0)}=45^{\circ}\right.$ ) with respect to the SNRs for the number of snapshots of 128 , (b) second incident signal $\left(\theta_{2}^{(0)}=65^{\circ}\right)$ with respect to the SNRs for the number of snapshots of 128 , (c) first incident signal $\left(\theta_{1}^{(0)}=45^{\circ}\right)$ with respect to the number of snapshots for $\mathrm{SNR}=-5 \mathrm{~dB}$, and $(\mathrm{d})$ second incident signal $\left(\theta_{2}^{(0)}=65^{\circ}\right)$ with respect to the number of snapshots for $\mathrm{SNR}=-5 \mathrm{~dB}$.

1 st, true $=-45$ deg., corr. $=1$, snapshots $=128$, repe. $=10000$, dist. $=0.5$ lam., step $=0.1 \mathrm{deg}$.

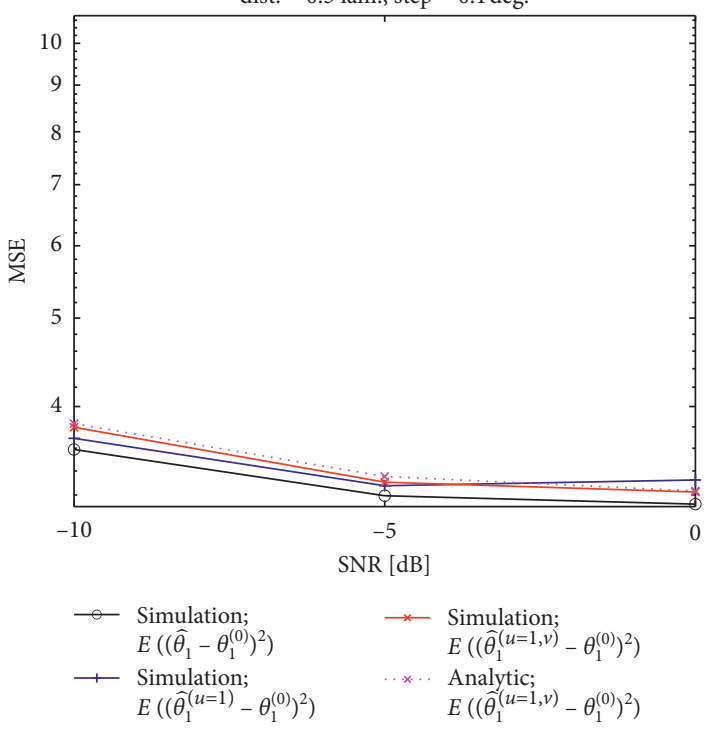

(a)

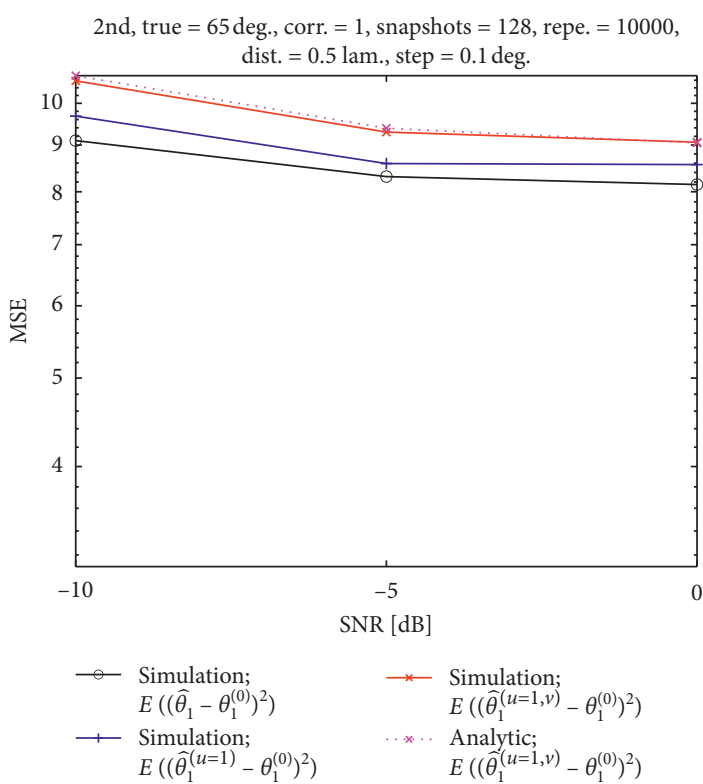

(b)

Figure 6: Continued. 


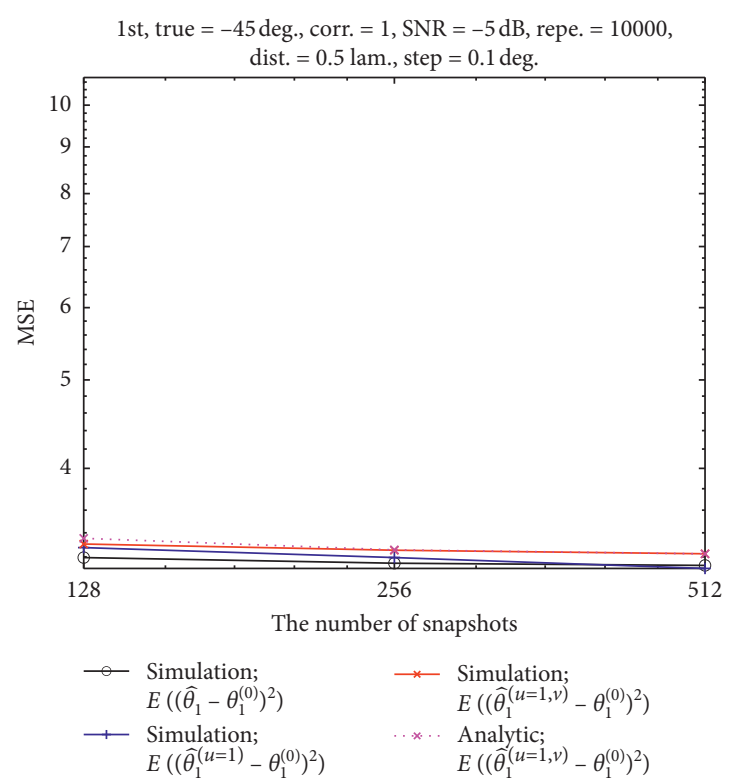

(c)

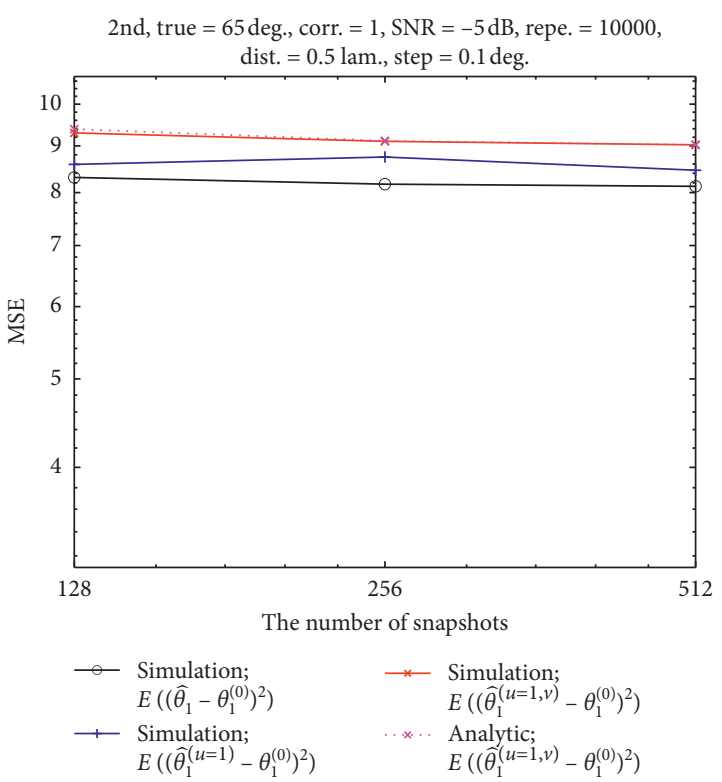

(d)

FIGURE 6: MSEs of analytic approach and that of Monte-Carlo simulation-based approach (coherent signals from $\theta_{1}^{(0)}=-45^{\circ}$ and $\theta_{2}^{(0)}=65^{\circ}$ ): (a) first incident signal $\left(\theta_{1}^{(0)}=-45^{\circ}\right)$ with respect to the SNRs for the number of snapshots of 128 , (b) second incident signal $\left(\theta_{2}^{(0)}=65^{\circ}\right)$ with respect to the SNRs for the number of snapshots of 128 , (c) first incident signal $\left(\theta_{1}^{(0)}=-45^{\circ}\right)$ with respect to the number of snapshots for $\mathrm{SNR}=-5 \mathrm{~dB}$, and $(\mathrm{d})$ second incident signal $\left(\theta_{2}^{(0)}=65^{\circ}\right)$ with respect to the number of snapshots for $\mathrm{SNR}=-5 \mathrm{~dB}$.

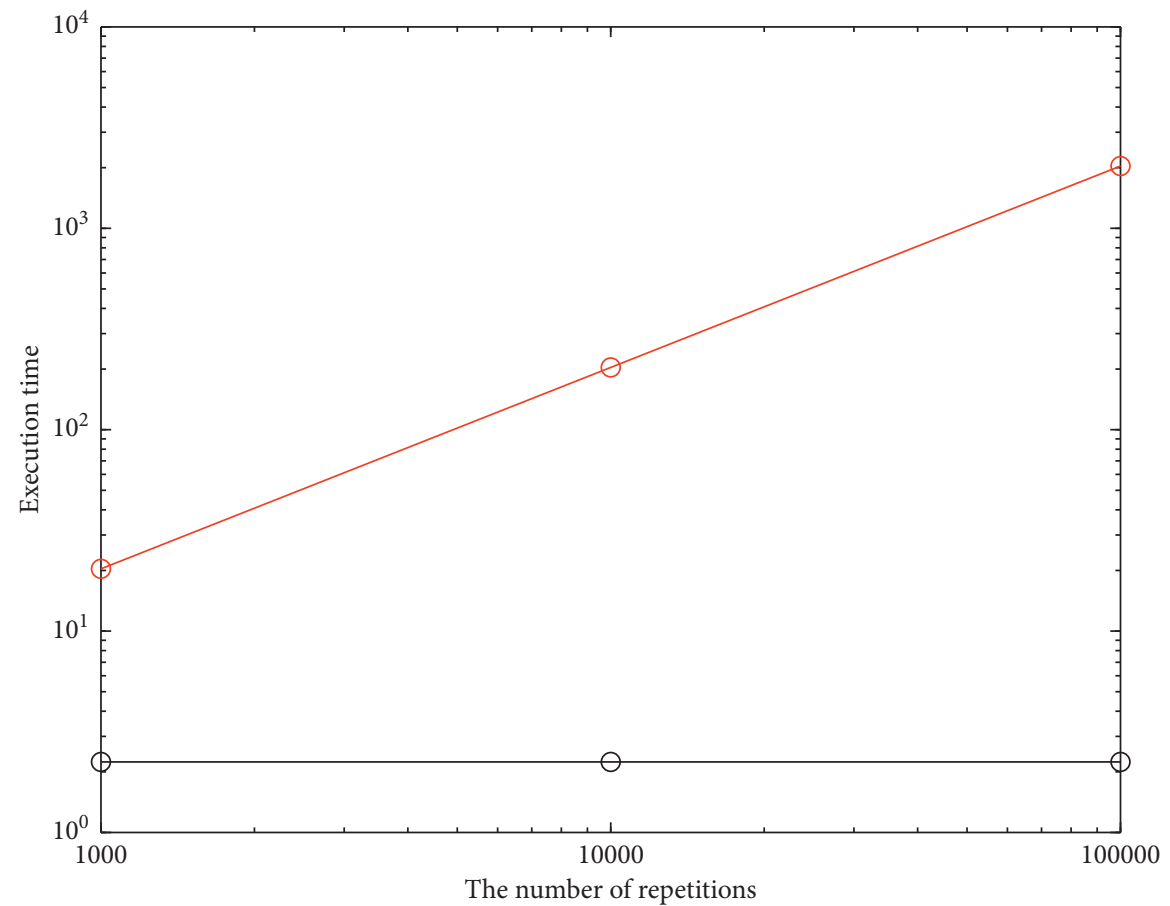

Figure 7: Execution time of analytic approach and that of Monte Carlo simulation-based approach with respect to the number of repetitions.

It is confirmed in Figure 5 that the agreements between the analytic MSEs and the empirical MSEs of $\hat{\theta}_{1}^{(u=1, v)}$. It is also shown in Figure 5 that the empirical MSEs of $\hat{\theta}_{1}^{(u=1)}$ and $\hat{\theta}_{1}^{\prime(u=1, v)}$ are close to those of $\hat{\theta}_{1}^{\prime}$.
5.3. Fully Correlated Incident Signals. In this section, fully correlated incident signals are considered. Fully correlated signals refer to coherent signals, which usually occur in the case of multipath environment. The correlation coefficient between two incident signals is set to be unity. The true 
incident angles are set to be $\theta_{1}^{(0)}=-45^{\circ}$ and $\theta_{2}^{(0)}=65^{\circ}$. In Figure 6 , the results for elemental distances of $0.5 \lambda$ are illustrated. It is shown in Figure 6 that the analytically obtained MSEs are close to simulation-based MSEs, which implies that the analytically obtained MSE can be used to see how accurate the simulation-based estimates in the conventional beamforming algorithm are.

5.4. Computational Complexity. Quantitative comparison of computation time is presented in Section 5.4. Note that the execution time is independent of whether the incident signals are correlated or not. In addition, the execution time is also independent of separation between the two incident signals. Therefore, the computation time is arbitrarily shown for the correlated closely spaced incident signals.

Figure 7 illustrates how computationally efficient the proposed algorithm is. The number of sensor is five, and the number of snapshots is 32 . The search step of the Monte Carlo simulation is $0.1 \mathrm{deg}$. The execution times of the simulation-based MSEs and analytic MSEs are illustrated with respect to the number of repetitions. Note that the execution time for analytically derived MSE is essentially independent of the number of repetitions since only one evaluation of expression (33) is necessary in the analytic approach. On the contrary, in the simulation-based approach, the empirical MSEs are obtained from 1000, 10,000, and 100,000 evaluations in (38). Therefore, the computational cost of the simulation-based performance analysis increases with the increase of the number of repetitions. Note that the number of repetitions, $T$, should be large to get a reliable MES estimate.

It is clearly shown in Figure 5(d) that execution time for the Monte Carlo simulation-based MSE is much greater than that for the analytically derived MSE even for the number of repetitions of xx. Figure 7 illustrates that getting analytically derived MSE is much less computationally intensive than getting Monte Carlo simulation-based MSE, which justifies why the analytically derived MSEs should be employed for performance analysis.

\section{Conclusions}

In this paper, we propose a new performance analysis of the conventional beamforming algorithm. For the conventional beamforming algorithm, we derive closed-form expressions of the estimates of the AOAs for small estimation error. The formulation accounts for various effects such as the finite number of snapshots, SNR, and the number of repetitions. The formulation is based on the assumption that when the estimation error is small, a cost function derived from the conventional beamforming algorithm can be approximated using the Taylor series expansion around the true incident direction. We derive closed-form expressions of the errors of the various estimates. In addition, closed-form expression of the MSE of one of the estimates is derived. All the derived expressions are validated using the numerical results and the CRBs.

The usefulness of the derived expression is that the MSEs of the conventional beamforming algorithm can be available from the derived expression without actually performing the Monte Carlo simulation, which is illustrated in the numerical results. By using the derived expression, we can get a quantitative measure of how accurate the estimates are without actually performing the Monte Carlo simulation.

It is shown in the numerical results that the proposed scheme works both for uncorrelated incident signals and coherent incident signals. Therefore, the computationally intensive empirical MSEs are very close to the computationally efficient analytic MSEs even for coherent incident signals, which imply that the analytically derived MSEs can be used to quantify the estimation accuracy of the conventional beamforming algorithm both for uncorrelated incident signals and coherent incident signals.

It is also illustrated that the proposed scheme is valid for the case of closely spaced incident signals. How closely incident signals can be handled in the conventional beamforming algorithm is highly dependent on the number of sensors. Once the incident signals can be resolved in the conventional beamforming algorithm, the proposed scheme can be employed to get the MSEs of the estimates analytically with much less computational complexity.

To avoid ambiguity, the elemental space between the uniform linear array should be no greater than $(\lambda / 2)$. In this paper, two cases of $0.5 \lambda$ and $0.3 \lambda$ are considered. The proposed scheme is validated for both elemental distances.

\section{Notation}
$(\cdot)^{T}:$
$(\cdot)^{-1}$ :
$(\cdot)$ :
$\left(\cdot^{\prime}\right)$ :
$\delta(\cdot)$ :

$M:$

$d:$

$L:$

$\mathbf{a}(\theta)$ :

$a_{i}(\theta)$ :

$\mathbf{k}_{n}=\left(\sin \theta_{n}, \cos \theta_{n}, 0\right)$ :

$\mathbf{z}_{m}=((m-1) \Delta, 0,0):$

$\psi_{m}(\theta):$

$\mathbf{x}\left(t_{i}\right):$

$\mathbf{x}^{\prime}\left(t_{i}\right)$ :
Transpose matrix

Inverse matrix

Noiseless quantity

Noisy quantity

Difference between the noisy quantity and the corresponding noiseless quantity

The number of sensors

The number of incident signals

The number of snapshots

Array vector associated with azimuth $\theta$

$i$ th entry of $\mathbf{a}(\theta)$

Unit vector describing the direction of the incident signal

Coordinate of the $m$ th antenna out of $M$ antennas

The phase at the $m$ th antenna for the incident from azimuth $\theta$ signal with reference to the origin at $(x=$ $0, y=0, z=0$ ) when there is no additive noise

The noiseless signals on the antenna elements due to all the incident signals

The noisy signals on the antenna elements due to all the incident signals 


\begin{tabular}{|c|c|c|c|}
\hline $\mathbf{x}^{(n)}\left(t_{i}\right):$ & $\begin{array}{l}\text { The noiseless signals on the antenna } \\
\text { elements due to the } n \text {th incident } \\
\text { signal }\end{array}$ & $(\cdot)^{(v)}:$ & $\begin{array}{l}\text { The } V \text {-approximation based on the } \\
\text { fact that the smallest term out of } \\
\text { four terms has been omitted }\end{array}$ \\
\hline$\hat{\mathbf{R}}:$ & $\begin{array}{l}\text { Sample covariance matrix } \\
\text { associated with } \mathbf{x}\left(t_{j}\right)\end{array}$ & $(\cdot)_{(t)}:$ & $\begin{array}{l}\text { The quantity associated with } t \text { th } \\
\text { repetition out of } T \text { repetitions. }\end{array}$ \\
\hline$\hat{R}_{k l}:$ & The entries at the $k$ th row and the & & \\
\hline$\hat{R}^{\prime}:$ & Sample covariance matrix & Appendix & \\
\hline$\hat{R}_{k l}^{\prime}:$ & $\begin{array}{l}\text { associated with } \mathbf{x}^{\prime}\left(t_{i}\right) \\
\text { The entries at the } k \text { th row and the } \\
l \text { th column of the } \hat{R}^{\prime} \\
\text { Sample covariance matrix }\end{array}$ & $\begin{array}{l}\text { A. Second- } \\
\text { Complex } \\
\text { Variance } 0\end{array}$ & $\begin{array}{l}\text { entral Moment of Zero-mean } \\
\text { Random Variable with }\end{array}$ \\
\hline
\end{tabular}

$\hat{R}^{(n)} \quad$ associated with $\mathbf{x}^{(n)}\left(t_{i}\right)$

$\hat{R}_{k l}^{(n)}: \quad \quad$ The entries at the $k$ th row and the lth column of the $\hat{R}^{(n)}$

$F(\cdot)$ : $\quad$ The cost function associated with $\hat{\mathbf{R}}$

$F^{\prime}(\cdot)$ : $\quad$ The cost function associated with $\hat{R}^{\prime}$

$F^{(n)}(\cdot)$ : The cost function associated with

$(\cdot)^{(u)}: \quad$ The $u$ th order $U$-approximation via the Taylor series
Depending on how $a, b, d$, and $e$ are related, we define four cases:

Case I: $a=b$ and $d=e$

Case II: $a \neq b$ and $d=e$

Case III: $a=b$ and $d \neq e$

Case IV: $a \neq b$ and $d \neq e$

(1) $E\left[n_{a}\left(t_{d}\right) n_{b}^{*}\left(t_{e}\right)\right]$ :

$$
\begin{aligned}
E\left[n_{a}\left(t_{d}\right) n_{b}^{*}\left(t_{e}\right)\right] & =E\left[\left\{\operatorname{Re}\left[n_{a}\left(t_{d}\right)\right]+j \operatorname{Im}\left[n_{a}\left(t_{d}\right)\right]\right\}\left\{\operatorname{Re}\left[n_{b}\left(t_{e}\right)\right]-j \operatorname{Im}\left[n_{b}\left(t_{e}\right)\right]\right\}\right] \\
& =E\left[\operatorname{Re}\left[n_{a}\left(t_{d}\right)\right] \operatorname{Re}\left[n_{b}\left(t_{e}\right)\right]-j \operatorname{Re}\left[n_{a}\left(t_{d}\right)\right] \operatorname{Im}\left[n_{b}\left(t_{e}\right)\right]+j \operatorname{Im}\left[n_{a}\left(t_{d}\right)\right] \operatorname{Re}\left[n_{b}\left(t_{e}\right)\right]+\operatorname{Im}\left[n_{a}\left(t_{d}\right)\right] \operatorname{Im}\left[n_{b}\left(t_{e}\right)\right]\right] .
\end{aligned}
$$

For Case I, $E\left[n_{a}\left(t_{d}\right) n_{b}^{*}\left(t_{e}\right)\right]$ is given by

$$
\begin{aligned}
E\left[n_{a}\left(t_{d}\right) n_{b}^{*}\left(t_{e}\right)\right]= & E\left[n_{a}\left(t_{d}\right) n_{a}^{*}\left(t_{d}\right)\right]=E\left[\operatorname{Re}\left[n_{a}\left(t_{d}\right)\right] \operatorname{Re}\left[n_{a}\left(t_{d}\right)\right]\right]+E\left[\operatorname{Im}\left[n_{a}\left(t_{d}\right)\right] \operatorname{Im}\left[n_{a}\left(t_{d}\right)\right]\right] \\
& -j E\left[\operatorname{Re}\left[n_{a}\left(t_{d}\right)\right] E \operatorname{Im}\left[n_{a}\left(t_{d}\right)\right]\right]+j E\left[\operatorname{Im}\left[n_{a}\left(t_{d}\right)\right] E \operatorname{Re}\left[n_{a}\left(t_{d}\right)\right]\right]=\frac{\sigma^{2}}{2}+\frac{\sigma^{2}}{2}-0+0=\sigma^{2} .
\end{aligned}
$$

Similarly, it can be shown that $E\left[n_{a}\left(t_{d}\right) n_{b}^{*}\left(t_{e}\right)\right]$ is identically zero for Cases II-IV:

$E\left[n_{a}\left(t_{d}\right) n_{b}^{*}\left(t_{e}\right)\right]=0, \quad$ for Case II $\sim$ Case IV.

Note that, in deriving (A.1)-(A.3), we used the fact that the real part and the imaginary part of the noise are independent and identically distributed with $N\left(0,\left(\sigma^{2} / 2\right)\right)$.

(2) $E\left[n_{a}^{*}\left(t_{d}\right) n_{b}^{*}\left(t_{e}\right)\right]$ :

Using the same algebraic manipulation used in evaluating $E\left[n_{a}\left(t_{d}\right) n_{b}^{*}\left(t_{e}\right)\right]$, it can be shown that $E\left[n_{a}^{*}\left(t_{d}\right) n_{b}^{*}\left(t_{e}\right)\right]$ is equal to zero for Cases I-IV:

$E\left[n_{a}^{*}\left(t_{d}\right) n_{b}^{*}\left(t_{e}\right)\right]=0, \quad$ for Case I $\sim$ Case IV.

(3) $E\left[n_{a}\left(t_{d}\right) n_{b}\left(t_{e}\right)\right]$ :
Using the same algebraic manipulation used in evaluating $E\left[n_{a}\left(t_{d}\right) n_{b}^{*}\left(t_{e}\right)\right]$, it can be shown that $E\left[n_{a}\left(t_{d}\right) n_{b}\left(t_{e}\right)\right]$ is equal to zero for Cases I-IV:

$E\left[n_{a}\left(t_{d}\right) n_{b}\left(t_{e}\right)\right]=0, \quad$ for Case I $\sim$ Case IV.

\section{B. Third-order Central Moment of Zero-mean Complex Gaussian Random Variable with Variance $\sigma^{2}$}

We define ten cases depending on how $a, b, c, d$, and $e$ are related:

Case I: $a=b$ and $b=c$ and $e=d$

Case II: $a=b$ and $b \neq c$ and $e=d$

Case III: $a \neq b$ and $b=c$ and $e=d$

Case IV: $a=c$ and $c \neq b$ and $e=d$ 
Case V: $a \neq b$ and $b \neq c$ and $e=d$

Case VI: $a=b$ and $b=c$ and $e \neq d$

Case VII: $a=b$ and $b \neq c$ and $e \neq d$

Case VIII: $a \neq b$ and $b=c$ and $e \neq d$
Case IX: $a=c$ and $c \neq b$ and $e \neq d$

Case X: $a \neq b$ and $b \neq c$ and $e \neq d$

(1) $E\left[n_{a}\left(t_{d}\right) n_{b}^{*}\left(t_{e}\right) n_{c}\left(t_{e}\right)\right]$ :

$$
\begin{aligned}
E\left[n_{a}\left(t_{d}\right) n_{b}^{*}\left(t_{e}\right) n_{c}\left(t_{e}\right)\right]= & E\left[\left(\operatorname{Re}\left[n_{a}\left(t_{d}\right)\right]+j \operatorname{Im}\left[n_{a}\left(t_{d}\right)\right]\right)\left(\operatorname{Re}\left[n_{b}\left(t_{e}\right)\right]-j \operatorname{Im}\left[n_{b}\left(t_{e}\right)\right]\right)\left(\operatorname{Re}\left[n_{c}\left(t_{e}\right)\right]+j \operatorname{Im}\left[n_{c}\left(t_{e}\right)\right]\right)\right] \\
= & E\left[\operatorname{Re}\left[n_{a}\left(t_{d}\right)\right] \operatorname{Re}\left[n_{b}\left(t_{e}\right)\right] \operatorname{Re}\left[n_{c}\left(t_{e}\right)\right]\right]+j E\left[\operatorname{Re}\left[n_{a}\left(t_{d}\right)\right] \operatorname{Re}\left[n_{b}\left(t_{e}\right)\right] \operatorname{Im}\left[n_{c}\left(t_{e}\right)\right]\right] \\
& -j E\left[\operatorname{Re}\left[n_{a}\left(t_{d}\right)\right] \operatorname{Im}\left[n_{b}\left(t_{e}\right)\right] \operatorname{Re}\left[n_{c}\left(t_{e}\right)\right]\right]+j E\left[\operatorname{Im}\left[n_{a}\left(t_{d}\right)\right] \operatorname{Re}\left[n_{b}\left(t_{e}\right)\right] \operatorname{Re}\left[n_{c}\left(t_{e}\right)\right]\right] \\
& +E\left[\operatorname{Re}\left[n_{a}\left(t_{d}\right)\right] \operatorname{Im}\left[n_{b}\left(t_{e}\right)\right] \operatorname{Im}\left[n_{c}\left(t_{e}\right)\right]\right]-E\left[\operatorname{Im}\left[n_{a}\left(t_{d}\right)\right] \operatorname{Re}\left[n_{b}\left(t_{e}\right)\right] \operatorname{Im}\left[n_{c}\left(t_{e}\right)\right]\right] \\
& +E\left[\operatorname{Im}\left[n_{a}\left(t_{d}\right)\right] \operatorname{Im}\left[n_{b}\left(t_{e}\right)\right] \operatorname{Re}\left[n_{c}\left(t_{e}\right)\right]\right]+j E\left[\operatorname{Im}\left[n_{a}\left(t_{d}\right)\right] \operatorname{Im}\left[n_{b}\left(t_{e}\right)\right] \operatorname{Im}\left[n_{c}\left(t_{e}\right)\right]\right] .
\end{aligned}
$$

$E\left[n_{a}\left(t_{d}\right) n_{b}^{*}\left(t_{e}\right) n_{c}\left(t_{e}\right)\right]$ for Case I is identically zero:

$$
\begin{aligned}
E\left[n_{a}\left(t_{d}\right) n_{b}^{*}\left(t_{e}\right) n_{c}\left(t_{e}\right)\right]= & E\left[n_{a}\left(t_{d}\right) n_{a}^{*}\left(t_{d}\right) n_{a}\left(t_{d}\right)\right)=E\left[\operatorname{Re}\left[n_{a}\left(t_{d}\right)\right] \operatorname{Re}\left[n_{a}\left(t_{d}\right)\right] \operatorname{Re}\left[n_{a}\left(t_{d}\right)\right]\right] \\
& +j E\left[\operatorname{Re}\left[n_{a}\left(t_{d}\right)\right] \operatorname{Re}\left[n_{a}\left(t_{d}\right)\right]\right] E\left[\operatorname{Im}\left[n_{a}\left(t_{d}\right)\right]\right] \\
& -j E\left[\operatorname{Re}\left[n_{a}\left(t_{d}\right)\right] \operatorname{Re}\left[n_{a}\left(t_{d}\right)\right]\right] E\left[\operatorname{Im}\left[n_{a}\left(t_{d}\right)\right]\right]+j E\left[\operatorname{Im}\left[n_{a}\left(t_{d}\right)\right]\right] E\left[\operatorname{Re}\left[n_{a}\left(t_{d}\right)\right] \operatorname{Re}\left[n_{a}\left(t_{d}\right)\right]\right] \\
& +E\left[\operatorname{Re}\left[n_{a}\left(t_{d}\right)\right]\right] E\left[\operatorname{Im}\left[n_{a}\left(t_{d}\right)\right] \operatorname{Im}\left[n_{a}\left(t_{d}\right)\right]\right] \\
& -E\left[\operatorname{Im}\left[n_{a}\left(t_{d}\right)\right] \operatorname{Im}\left[n_{a}\left(t_{d}\right)\right]\right] E\left[\operatorname{Re}\left[n_{a}\left(t_{d}\right)\right]\right]+E\left[\operatorname{Im}\left[n_{a}\left(t_{d}\right)\right] \operatorname{Im}\left[n_{a}\left(t_{d}\right)\right]\right] E\left[\operatorname{Re}\left[n_{a}\left(t_{d}\right)\right]\right] \\
& +j E\left[\operatorname{Im}\left[n_{a}\left(t_{d}\right)\right] \operatorname{Im}\left[n_{a}\left(t_{d}\right)\right] \operatorname{Im}\left[n_{a}\left(t_{d}\right)\right]\right] \\
= & 0+j\left(\frac{\sigma^{2}}{2}\right) \times 0-j\left(\frac{\sigma^{2}}{2}\right) \times 0+j\left(\frac{\sigma^{2}}{2}\right) \times 0+\left(\frac{\sigma^{2}}{2}\right) \times 0-\left(\frac{\sigma^{2}}{2}\right) \times 0+\left(\frac{\sigma^{2}}{2}\right) \times 0+j \times 0=0 .
\end{aligned}
$$

Note that, in deriving (B.1) and (B.2), we used the fact that the real part and the imaginary part of noise are independent and identically distributed with $N\left(0,\left(\sigma^{2} / 2\right)\right)$.

Using the same manipulation used in obtaining (B.2), it can be shown that $E\left[n_{a}\left(t_{d}\right) n_{b}^{*}\left(t_{e}\right) n_{c}\left(t_{e}\right)\right]$ is identically zero for Cases II-X:
$E\left[n_{a}\left(t_{d}\right) n_{b}^{*}\left(t_{e}\right) n_{c}\left(t_{e}\right)\right]=0 \quad$ for Case II - Case X.

From (B.2) and (B.3), $E\left[n_{a}\left(t_{d}\right) n_{b}^{*}\left(t_{e}\right) n_{c}\left(t_{e}\right)\right]$ is zero for all ten cases defined in Cases I-X:

$E\left[n_{a}\left(t_{d}\right) n_{b}^{*}\left(t_{e}\right) n_{c}\left(t_{e}\right)\right]=0, \quad$ for Case I - Case X.

(2) $E\left[n_{a}^{*}\left(t_{d}\right) n_{b}^{*}\left(t_{e}\right) n_{c}\left(t_{e}\right)\right]$ :

$$
\begin{aligned}
E\left[n_{a}^{*}\left(t_{d}\right) n_{b}^{*}\left(t_{e}\right) n_{c}\left(t_{e}\right)\right]= & E\left[\left(\operatorname{Re}\left[n_{a}\left(t_{d}\right)\right]-j \operatorname{Im}\left[n_{a}\left(t_{d}\right)\right]\right)\left(\operatorname{Re}\left[n_{b}\left(t_{e}\right)\right]-j \operatorname{Im}\left[n_{b}\left(t_{e}\right)\right]\right)\left(\operatorname{Re}\left[n_{e}\left(t_{e}\right)\right]-j \operatorname{Im}\left[n_{c}\left(t_{e}\right)\right]\right)\right] \\
= & E\left[\operatorname{Re}\left[n_{a}\left(t_{d}\right)\right] \operatorname{Re}\left[n_{b}\left(t_{e}\right)\right] \operatorname{Re}\left[n_{c}\left(t_{e}\right)\right]\right]+j E\left[\operatorname{Re}\left[n_{a}\left(t_{d}\right)\right] \operatorname{Re}\left[n_{b}\left(t_{e}\right)\right] \operatorname{Im}\left[n_{c}\left(t_{e}\right)\right]\right] \\
& -j E\left[\operatorname{Re}\left[n_{a}\left(t_{d}\right)\right] \operatorname{Im}\left[n_{b}\left(t_{e}\right)\right] \operatorname{Re}\left[n_{c}\left(t_{e}\right)\right]\right]-j E\left[\operatorname{Im}\left[n_{a}\left(t_{d}\right)\right] \operatorname{Re}\left[n_{b}\left(t_{e}\right)\right] \operatorname{Re}\left[n_{c}\left(t_{e}\right)\right]\right] \\
& +E\left[\operatorname{Re}\left[n_{a}\left(t_{d}\right)\right] \operatorname{Im}\left[n_{b}\left(t_{e}\right)\right] \operatorname{Im}\left[n_{c}\left(t_{e}\right)\right]\right]+E\left[\operatorname{Im}\left[n_{a}\left(t_{d}\right)\right] \operatorname{Re}\left[n_{b}\left(t_{e}\right)\right] \operatorname{Im}\left[n_{c}\left(t_{e}\right)\right]\right] \\
& -E\left[\operatorname{Im}\left[n_{a}\left(t_{d}\right)\right] \operatorname{Im}\left[n_{b}\left(t_{e}\right)\right] \operatorname{Re}\left[n_{c}\left(t_{e}\right)\right]\right]-j E\left[\operatorname{Im}\left[n_{a}\left(t_{d}\right)\right] \operatorname{Im}\left[n_{b}\left(t_{e}\right)\right] \operatorname{Im}\left[n_{c}\left(t_{e}\right)\right]\right]
\end{aligned}
$$


In a similar way to obtain (B.4), we obtain

$E\left[n_{a}^{*}\left(t_{d}\right) n_{b}^{*}\left(t_{e}\right) n_{c}\left(t_{e}\right)\right]=0$ for Case I - Case X.

\section{Fourth-order Central Moment of Zero-mean Complex Gaussian Random Variable with Variance $\sigma^{2}$}

$$
\begin{aligned}
E & {\left[n_{k}\left(t_{i}\right) n_{l}^{*}\left(t_{i}\right) n_{k^{\prime}}^{*}\left(t_{i^{\prime}}\right) n_{l^{\prime}}\left(t_{i^{\prime}}\right)\right] } \\
= & E\left[\left\{\operatorname{Re}\left[n_{k}\left(t_{i}\right)\right]+j \operatorname{Im}\left[n_{k}\left(t_{i}\right)\right]\right\}\left\{\operatorname{Re}\left[n_{l}\left(t_{i}\right)\right]-j \operatorname{Im}\left[n_{l}\left(t_{i}\right)\right]\right\}\right. \\
& \left.\cdot\left\{\operatorname{Re}\left[n_{k^{\prime}}\left(t_{i^{\prime}}\right)\right]+j \operatorname{Im}\left[n_{k^{\prime}}\left(t_{i^{\prime}}\right)\right]\right\}\left\{\operatorname{Re}\left[n_{l^{\prime}}\left(t_{i}\right)\right]+j \operatorname{Im}\left[n_{l^{\prime}}\left(t_{i}\right)\right]\right\}\right] .
\end{aligned}
$$

(a) $i=i^{\prime}$

For $i=i^{\prime}$, (C.1) can be written as

$$
\begin{aligned}
& \sum_{l=1}^{M} \sum_{k=1}^{M}\left(f_{k l}\left(\theta_{n}^{(0)}\right) \hat{R}_{k l}^{(n)}\right)+\sum_{l=1}^{M} \sum_{k=1}^{M}\left(f_{k l}\left(\theta_{n}^{(0)}\right) \delta \hat{R}_{k l}^{(n)}\right) \\
& +\delta \theta_{n}^{(u=1)} \sum_{l=1}^{M} \sum_{k=1}^{M}\left(\left.\frac{\mathrm{d}}{\mathrm{d} \theta} f_{k l}(\theta)\right|_{\left.\theta=\theta_{n}^{(0)} \hat{R}_{k l}^{(n)}\right)}\right. \\
& +\delta \theta_{n}^{(u=1)} \sum_{l=1}^{M} \sum_{k=1}^{M}\left(\left.\frac{\mathrm{d}}{\mathrm{d} \theta} f_{k l}(\theta)\right|_{\left.\theta=\theta_{n}^{(0)} \delta \hat{R}_{k l}^{(n)}\right)=0 .}\right.
\end{aligned}
$$

The first term of (C.2) is given by (C.4), where the results in Appendix A and Appendix B are used:

$$
\begin{aligned}
& E\left[\operatorname{Re}\left[n_{k}\left(t_{i}\right)\right] \operatorname{Re}\left[n_{l}\left(t_{i}\right)\right] \operatorname{Re}\left[n_{k^{\prime}}\left(t_{i}\right)\right] \operatorname{Re}\left[n_{l^{\prime}}\left(t_{i}\right)\right]\right]
\end{aligned}
$$

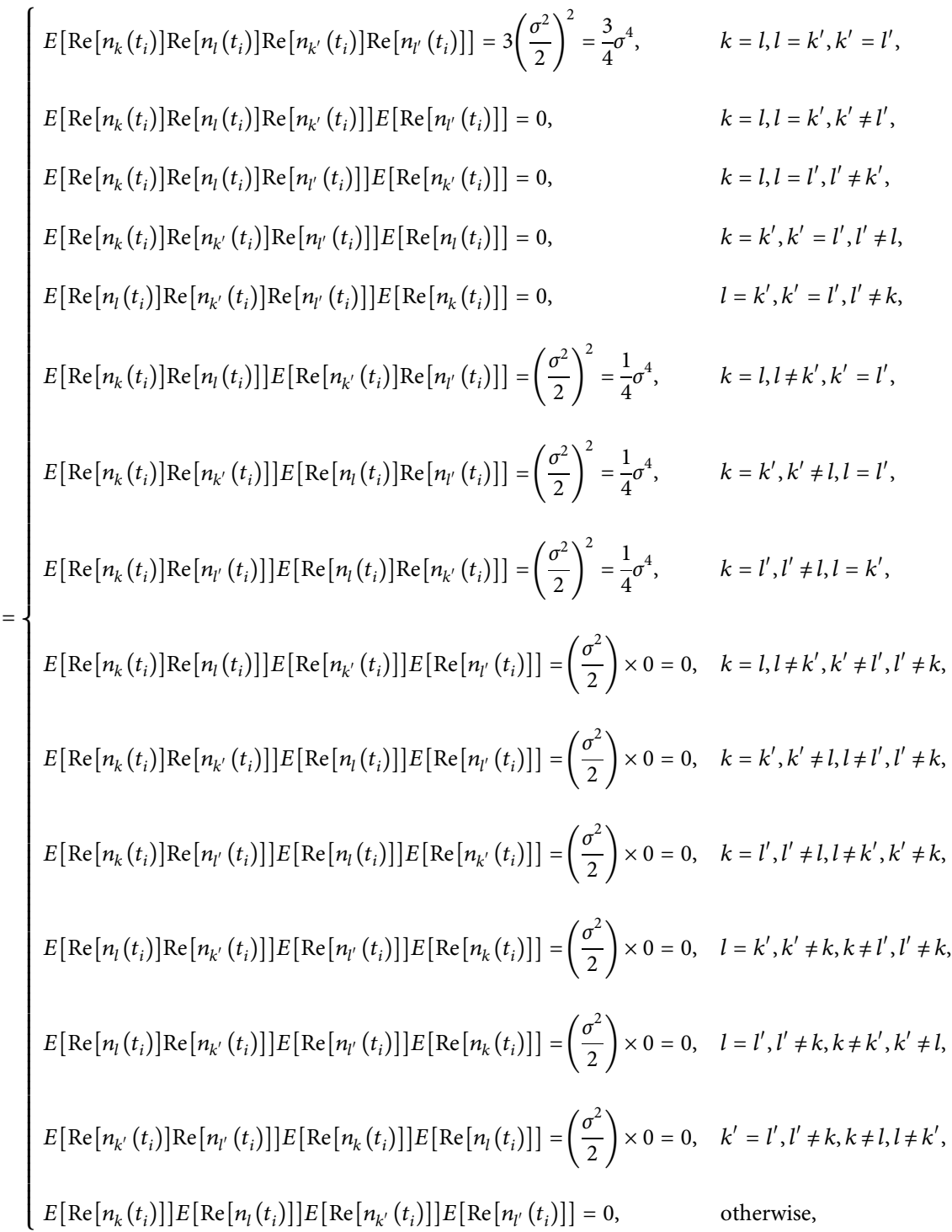


$E\left[\operatorname{Re}\left[n_{k}\left(t_{i}\right)\right] \operatorname{Re}\left[n_{l}\left(t_{i}\right)\right]\right] E\left[\operatorname{Im}\left[n_{k^{\prime}}\left(t_{i}\right)\right] \operatorname{Im}\left[n_{l^{\prime}}\left(t_{i}\right)\right]\right]$

$$
= \begin{cases}E\left[\operatorname{Re}\left[n_{k}\left(t_{i}\right)\right] \operatorname{Re}\left[n_{l}\left(t_{i}\right)\right]\right] E\left[\operatorname{Im}\left[n_{k^{\prime}}\left(t_{i}\right)\right] \operatorname{Im}\left[n_{l^{\prime}}\left(t_{i}\right)\right]\right]=\frac{1}{4} \sigma^{4}, & k=l, l=k^{\prime}, k^{\prime}=l^{\prime}, \\ E\left[\operatorname{Re}\left[n_{k}\left(t_{i}\right)\right] \operatorname{Re}\left[n_{l}\left(t_{i}\right)\right]\right] E\left[\operatorname{Im}\left[n_{k^{\prime}}\left(t_{i}\right)\right] \operatorname{Im}\left[n_{l^{\prime}}\left(t_{i}\right)\right]\right]=\frac{1}{4} \sigma^{4}, & k=l, l \neq k^{\prime}, k^{\prime}=l^{\prime}, \\ E\left[\operatorname{Re}\left[n_{k}\left(t_{i}\right)\right] \operatorname{Re}\left[n_{l}\left(t_{i}\right)\right]\right] E\left[\operatorname{Im}\left[n_{k^{\prime}}\left(t_{i}\right)\right] \operatorname{Im}\left[n_{l^{\prime}}\left(t_{i}\right)\right]\right]=0, & \text { otherwise, }\end{cases}
$$

$E\left[\operatorname{Re}\left[n_{k}\left(t_{i}\right)\right] \operatorname{Re}\left[n_{l^{\prime}}\left(t_{i}\right)\right]\right] E\left[\operatorname{Im}\left[n_{l}\left(t_{i}\right)\right] \operatorname{Im}\left[n_{k^{\prime}}\left(t_{i}\right)\right]\right]$

$$
=\left\{\begin{array}{l}
E\left[\operatorname{Re}\left[n_{k}\left(t_{i}\right)\right] \operatorname{Re}\left[n_{l^{\prime}}\left(t_{i}\right)\right]\right] E\left[\operatorname{Im}\left[n_{l}\left(t_{i}\right)\right] \operatorname{Im}\left[n_{k^{\prime}}\left(t_{i}\right)\right]\right]=\frac{1}{4} \sigma^{4}, \quad k=l, l=k^{\prime}, k^{\prime}=l^{\prime}, \\
E\left[\operatorname{Re}\left[n_{k}\left(t_{i}\right)\right] \operatorname{Re}\left[n_{l^{\prime}}\left(t_{i}\right)\right]\right] E\left[\operatorname{Im}\left[n_{l}\left(t_{i}\right)\right] \operatorname{Im}\left[n_{k^{\prime}}\left(t_{i}\right)\right]\right]=\frac{1}{4} \sigma^{4}, \quad k=l^{\prime}, l^{\prime} \neq l, l=k^{\prime}, \\
E\left[\operatorname{Re}\left[n_{k}\left(t_{i}\right)\right] \operatorname{Re}\left[n_{l^{\prime}}\left(t_{i}\right)\right]\right] E\left[\operatorname{Im}\left[n_{l}\left(t_{i}\right)\right] \operatorname{Im}\left[n_{k^{\prime}}\left(t_{i}\right)\right]\right]=0, \quad \text { otherwise, }
\end{array}\right.
$$

$E\left[\operatorname{Im}\left[n_{k}\left(t_{i}\right)\right] \operatorname{Im}\left[n_{l}\left(t_{i}\right)\right]\right] E\left[\operatorname{Re}\left[n_{k^{\prime}}\left(t_{i}\right)\right] \operatorname{Re}\left[n_{l^{\prime}}\left(t_{i}\right)\right]\right]$

$$
= \begin{cases}E\left[\operatorname{Im}\left[n_{k}\left(t_{i}\right)\right] \operatorname{Im}\left[n_{l}\left(t_{i}\right)\right]\right] E\left[\operatorname{Re}\left[n_{k^{\prime}}\left(t_{i}\right)\right] \operatorname{Re}\left[n_{l^{\prime}}\left(t_{i}\right)\right]\right]=\frac{1}{4} \sigma^{4}, & k=l, l=k^{\prime}, k^{\prime}=l^{\prime}, \\ E\left[\operatorname{Im}\left[n_{k}\left(t_{i}\right)\right] \operatorname{Im}\left[n_{l}\left(t_{i}\right)\right]\right] E\left[\operatorname{Re}\left[n_{k^{\prime}}\left(t_{i}\right)\right] \operatorname{Re}\left[n_{l^{\prime}}\left(t_{i}\right)\right]\right]=\frac{1}{4} \sigma^{4}, & k=l, l \neq k^{\prime}, k^{\prime}=l^{\prime}, \\ E\left[\operatorname{Im}\left[n_{k}\left(t_{i}\right)\right] \operatorname{Im}\left[n_{l}\left(t_{i}\right)\right]\right] E\left[\operatorname{Re}\left[n_{k^{\prime}}\left(t_{i}\right)\right] \operatorname{Re}\left[n_{l^{\prime}}\left(t_{i}\right)\right]\right]=0, & \text { otherwise, }\end{cases}
$$

$E\left[\operatorname{Re}\left[n_{l}\left(t_{i}\right)\right] \operatorname{Re}\left[n_{l^{\prime}}\left(t_{i}\right)\right]\right] E\left[\operatorname{Im}\left[n_{k}\left(t_{i}\right)\right] \operatorname{Im}\left[n_{k^{\prime}}\left(t_{i}\right)\right]\right]$

$$
= \begin{cases}E\left[\operatorname{Re}\left[n_{l}\left(t_{i}\right)\right] \operatorname{Re}\left[n_{l^{\prime}}\left(t_{i}\right)\right]\right] E\left[\operatorname{Im}\left[n_{k}\left(t_{i}\right)\right] \operatorname{Im}\left[n_{k^{\prime}}\left(t_{i}\right)\right]\right]=\frac{1}{4} \sigma^{4}, & k=l, l=k^{\prime}, k^{\prime}=l^{\prime}, \\ E\left[\operatorname{Re}\left[n_{l}\left(t_{i}\right)\right] \operatorname{Re}\left[n_{l^{\prime}}\left(t_{i}\right)\right]\right] E\left[\operatorname{Im}\left[n_{k}\left(t_{i}\right)\right] \operatorname{Im}\left[n_{k^{\prime}}\left(t_{i}\right)\right]\right]=\frac{1}{4} \sigma^{4}, & k=k^{\prime}, k^{\prime} \neq l, l=l^{\prime}, \\ E\left[\operatorname{Re}\left[n_{l}\left(t_{i}\right)\right] \operatorname{Re}\left[n_{l^{\prime}}\left(t_{i}\right)\right]\right] E\left[\operatorname{Im}\left[n_{k}\left(t_{i}\right)\right] \operatorname{Im}\left[n_{k^{\prime}}\left(t_{i}\right)\right]\right]=0, & \text { otherwise, }\end{cases}
$$

$E\left[\operatorname{Re}\left[n_{l}\left(t_{i}\right)\right] \operatorname{Re}\left[n_{k^{\prime}}\left(t_{i}\right)\right]\right] E\left[\operatorname{Im}\left[n_{k}\left(t_{i}\right)\right] \operatorname{Im}\left[n_{l^{\prime}}\left(t_{i}\right)\right]\right]$

$$
= \begin{cases}E\left[\operatorname{Re}\left[n_{l}\left(t_{i}\right)\right] \operatorname{Re}\left[n_{k^{\prime}}\left(t_{i}\right)\right]\right] E\left[\operatorname{Im}\left[n_{k}\left(t_{i}\right)\right] \operatorname{Im}\left[n_{l^{\prime}}\left(t_{i}\right)\right]\right]=\frac{1}{4} \sigma^{4}, \quad k=l, l=k^{\prime}, k^{\prime}=l^{\prime}, \\ E\left[\operatorname{Re}\left[n_{l}\left(t_{i}\right)\right] \operatorname{Re}\left[n_{k^{\prime}}\left(t_{i}\right)\right]\right] E\left[\operatorname{Im}\left[n_{k}\left(t_{i}\right)\right] \operatorname{Im}\left[n_{l^{\prime}}\left(t_{i}\right)\right]\right]=\frac{1}{4} \sigma^{4}, \quad k=l^{\prime}, l^{\prime} \neq l, l=k^{\prime}, \\ E\left[\operatorname{Re}\left[n_{l}\left(t_{i}\right)\right] \operatorname{Re}\left[n_{k^{\prime}}\left(t_{i}\right)\right]\right] E\left[\operatorname{Im}\left[n_{k}\left(t_{i}\right)\right] \operatorname{Im}\left[n_{l^{\prime}}\left(t_{i}\right)\right]\right]=0, & \text { otherwise, }\end{cases}
$$




$$
\begin{aligned}
& E\left[\operatorname{Re}\left[n_{k}\left(t_{i}\right)\right] \operatorname{Re}\left[n_{k^{\prime}}\left(t_{i}\right)\right]\right] E\left[\operatorname{Im}\left[n_{l}\left(t_{i}\right)\right] \operatorname{Im}\left[n_{l^{\prime}}\left(t_{i}\right)\right]\right] \\
& = \begin{cases}E\left[\operatorname{Re}\left[n_{k}\left(t_{i}\right)\right] \operatorname{Re}\left[n_{k^{\prime}}\left(t_{i}\right)\right]\right] E\left[\operatorname{Im}\left[n_{l}\left(t_{i}\right)\right] \operatorname{Im}\left[n_{l^{\prime}}\left(t_{i}\right)\right]\right]=\frac{1}{4} \sigma^{4}, & k=l, l=k^{\prime}, k^{\prime}=l^{\prime}, \\
E\left[\operatorname{Re}\left[n_{k}\left(t_{i}\right)\right] \operatorname{Re}\left[n_{k^{\prime}}\left(t_{i}\right)\right]\right] E\left[\operatorname{Im}\left[n_{l}\left(t_{i}\right)\right] \operatorname{Im}\left[n_{l^{\prime}}\left(t_{i}\right)\right]\right]=\frac{1}{4} \sigma^{4}, & k=k^{\prime}, k^{\prime} \neq l, l=l^{\prime}, \\
E\left[\operatorname{Re}\left[n_{k}\left(t_{i}\right)\right] \operatorname{Re}\left[n_{k^{\prime}}\left(t_{i}\right)\right]\right] E\left[\operatorname{Im}\left[n_{l}\left(t_{i}\right)\right] \operatorname{Im}\left[n_{l^{\prime}}\left(t_{i}\right)\right]\right]=0, & \text { otherwise, }\end{cases} \\
& E\left[\operatorname{Im}\left[n_{k}\left(t_{i}\right)\right] \operatorname{Im}\left[n_{l}\left(t_{i}\right)\right] \operatorname{Im}\left[n_{k_{l}}\left(t_{i}\right)\right] \operatorname{Im}\left[n_{l}\left(t_{i}\right)\right]\right] \\
& = \begin{cases}E\left[\operatorname{Im}\left[n_{k}\left(t_{i}\right)\right] \operatorname{Im}\left[n_{l}\left(t_{i}\right)\right] \operatorname{Im}\left[n_{k_{\prime}}\left(t_{i}\right)\right] \operatorname{Im}\left[n_{l_{l}}\left(t_{i}\right)\right]\right]=\frac{3}{4} \sigma^{4}, & k=l, l=k \prime, k \prime=l \prime, \\
E\left[\operatorname{Im}\left[n_{k}\left(t_{i}\right)\right] \operatorname{Im}\left[n_{l}\left(t_{i}\right)\right]\right] E\left[\operatorname{Im}\left[n_{k_{l}}\left(t_{i}\right)\right] \operatorname{Im}\left[n_{l,}\left(t_{i}\right)\right]\right]=\frac{1}{4} \sigma^{4}, & k=l, l \neq k \prime, k \prime=l \prime, \\
E\left[\operatorname{Im}\left[n_{k}\left(t_{i}\right)\right] \operatorname{Im}\left[n_{k_{l}}\left(t_{i}\right)\right]\right] E\left[\operatorname{Im}\left[n_{l}\left(t_{i}\right)\right] \operatorname{Im}\left[n_{l,}\left(t_{i}\right)\right]\right]=\frac{1}{4} \sigma^{4}, & k=k \prime, k \prime \neq l, l=l \prime, \\
E\left[\operatorname{Im}\left[n_{k}\left(t_{i}\right)\right] \operatorname{Im}\left[n_{l,}\left(t_{i}\right)\right]\right] E\left[\operatorname{Im}\left[n_{l}\left(t_{i}\right)\right] \operatorname{Im}\left[n_{k_{l}}\left(t_{i}\right)\right]\right]=\frac{1}{4} \sigma^{4}, & k=l \prime, l \prime \neq l, l=k \prime, \\
E\left[\operatorname{Im}\left[n_{k}\left(t_{i}\right)\right] \operatorname{Im}\left[n_{l}\left(t_{i}\right)\right] \operatorname{Im}\left[n_{k_{l}}\left(t_{i}\right)\right] \operatorname{Im}\left[n_{l}\left(t_{i}\right)\right]\right]=0, & \text { otherwise, }\end{cases} \\
& E\left[n_{k}\left(t_{i}\right) n_{l}^{*}\left(t_{i}\right) n_{k}^{*}\left(t_{i}\right) n_{l}\left(t_{i}\right)\right] \\
& = \begin{cases}\frac{3}{4} \sigma^{4}+\frac{1}{4} \sigma^{4}-\frac{1}{4} \sigma^{4}+\frac{1}{4} \sigma^{4}+\frac{1}{4} \sigma^{4}-\frac{1}{4} \sigma^{4}+\frac{1}{4} \sigma^{4}+\frac{3}{4} \sigma^{4}=2 \sigma^{4}, & k=l, l=k \prime, k \prime=l \prime, \\
\frac{1}{4} \sigma^{4}+\frac{1}{4} \sigma^{4}+\frac{1}{4} \sigma^{4}+\frac{1}{4} \sigma^{4}=\sigma^{4}, & k=l, l \neq k \prime, k \prime=l \prime, \\
\frac{1}{4} \sigma^{4}+\frac{1}{4} \sigma^{4}+\frac{1}{4} \sigma^{4}+\frac{1}{4} \sigma^{4}=\sigma^{4}, & k=k \prime, k \prime \neq l, l=l \prime, \\
0, & \text { otherwise. }\end{cases}
\end{aligned}
$$

Using the same scheme in getting (C.4), the other terms of (C.2) are given by (C.4)-(C.11). From
(C.4)-(C.10), $E\left[n_{k}\left(t_{i}\right) n_{l}^{*}\left(t_{i}\right) n_{k}^{*}\left(t_{i}\right) n_{l}\left(t_{i}\right)\right]$ in (C.2) is given by (C.11). 
(b) $i \neq i \prime$ :

$$
\begin{aligned}
E\left[n_{k}\left(t_{i}\right) n_{l}^{*}\left(t_{i}\right) n_{k}^{*}\left(t_{i_{1}}\right) n_{l_{l}}\left(t_{i_{1}}\right)\right]= & E\left[\operatorname{Re}\left[n_{k}\left(t_{i}\right)\right] \operatorname{Re}\left[n_{l}\left(t_{i}\right)\right] \operatorname{Re}\left[n_{k_{l}}\left(t_{i_{1}}\right)\right] \operatorname{Re}\left[n_{l_{l}}\left(t_{i_{1}}\right)\right]\right] \\
& +E\left[\operatorname{Re}\left[n_{k}\left(t_{i}\right)\right] \operatorname{Re}\left[n_{l}\left(t_{i}\right)\right] \operatorname{Im}\left[n_{k_{l}}\left(t_{i_{1}}\right)\right] \operatorname{Im}\left[n_{l_{l}}\left(t_{i_{1}}\right)\right]\right] \\
& +E\left[\operatorname{Im}\left[n_{k}\left(t_{i}\right)\right] \operatorname{Im}\left[n_{l}\left(t_{i}\right)\right] \operatorname{Re}\left[n_{k_{l}}\left(t_{i_{1}}\right)\right] \operatorname{Re}\left[n_{l_{l}}\left(t_{i_{1}}\right)\right]\right] \\
& +E\left[\operatorname{Im}\left[n_{k}\left(t_{i}\right)\right] \operatorname{Im}\left[n_{l}\left(t_{i}\right)\right] \operatorname{Im}\left[n_{k_{l}}\left(t_{i_{1}}\right)\right] \operatorname{Im}\left[n_{l_{l}}\left(t_{i_{1}}\right)\right]\right] .
\end{aligned}
$$

Using a similar way to get (C.11), for $i \neq i$,

$E\left[n_{k}\left(t_{i}\right) n_{l}^{*}\left(t_{i}\right) n_{k}^{*}\left(t_{i}\right) n_{l}\left(t_{i}\right)\right]$ is given by

$$
E\left[n_{k}\left(t_{i}\right) n_{l}^{*}\left(t_{i}\right) n_{k}^{*}\left(t_{i_{1}}\right) n_{l}\left(t_{i_{1}}\right)\right]= \begin{cases}\frac{1}{4} \sigma^{4}+\frac{1}{4} \sigma^{4}+\frac{1}{4} \sigma^{4}+\frac{1}{4} \sigma^{4}=\sigma^{4}, & \left(k=l \text { and } l=k^{\prime} \text { and } k^{\prime}=l^{\prime}\right), \\ \frac{1}{4} \sigma^{4}+\frac{1}{4} \sigma^{4}+\frac{1}{4} \sigma^{4}+\frac{1}{4} \sigma^{4}=\sigma^{4}, & \left(k=l \text { and } l \neq k^{\prime} \text { and } k^{\prime}=l^{\prime}\right) \\ 0, & \text { (otherwise) } .\end{cases}
$$

D. Derivation of $E\left[\boldsymbol{\delta} \widehat{R}_{l k} \delta \widehat{R}_{l^{\prime} k^{\prime}}^{*}\right]$ :

$$
\begin{aligned}
\hat{R}_{k l}^{\prime}= & \frac{1}{L} \sum_{i=1}^{L} x_{k} t_{i}+y_{k} t_{i}+n_{k} t_{i} x_{l}^{*} t_{i}+y_{l}^{*} t_{i}+n_{l}^{*} t_{i} \\
= & \frac{1}{L} \sum_{i=1}^{L} x_{k} t_{i} x_{l}^{*} t_{i}+x_{k} t_{i} y_{l}^{*} t_{i}+y_{k} t_{i} x_{l}^{*} t_{i} \\
& +y_{k} t_{i} y_{l}^{*} t_{i}+x_{k} t_{i}+y_{k} t_{i} n_{l}^{*} t_{i}+n_{k} t_{i} x_{l}^{*} t_{i}+y_{l}^{*} t_{i}+n_{k} t_{i} n_{l}^{*} t_{i}, \\
\hat{R}_{k l}= & \frac{1}{L} \sum_{i=1}^{L} x_{k} t_{i} x_{l}^{*} t_{i}, \\
\delta \hat{R}_{k l}= & \frac{1}{L} \sum_{i=1}^{L} x_{k} t_{i} y_{l}^{*} t_{i}+y_{k} t_{i} x_{l}^{*} t_{i}+y_{k} t_{i} y_{l}^{*} t_{i} \\
& +x_{k} t_{i}+y_{k} t_{i} n_{l}^{*} t_{i}+n_{k} t_{i} x_{l}^{*} t_{i}+y_{l}^{*} t_{i}+n_{k} t_{i} n_{l}^{*} t_{i} .
\end{aligned}
$$

From (D.3), due to the fact that noiseless signals are not stochastic and that the real part and the imaginary part of noise are zero-mean Gaussian distributed with variance $\left(\sigma^{2} / 2\right), E\left[\delta \widehat{R}_{k l} \delta \widehat{R}_{k^{\prime} l}^{*}\right]$ is given by 


$$
\begin{aligned}
& E\left[\delta \hat{R}_{k l} \delta \hat{R}_{k^{\prime} l^{\prime}}^{*}\right]=\frac{1}{L^{2}} \sum_{i=1}^{L} \sum_{i^{\prime}=1}^{L} x_{k} t_{i} y_{l}^{*} t_{i} x_{k^{\prime}}^{*} t_{i}^{\prime} y_{l^{\prime}} t_{i}^{\prime} \\
& +x_{k} t_{i} y_{l}^{*} t_{i} y_{k^{\prime}}^{*} t_{i^{\prime}} x_{l^{\prime}} t_{i^{\prime}}+x_{k} t_{i} y_{l}^{*} t_{i} y_{k^{\prime}}^{*} t_{i^{\prime}} y_{l^{\prime}} t_{i^{\prime}}+y_{k} t_{i} x_{l}^{*} t_{i} x_{k^{\prime}}^{*} t_{i^{\prime}} y_{l^{\prime}} t_{i^{\prime}} \\
& +y_{k} t_{i} x_{l}^{*} t_{i} y_{k^{\prime}}^{*} t_{i^{\prime}} x_{l^{\prime}} t_{i^{\prime}}+y_{k} t_{i} x_{l}^{*} t_{i} y_{k^{\prime}}^{*} t_{i}^{\prime} y_{l^{\prime}} t_{i^{\prime}}+y_{k} t_{i} y_{l}^{*} t_{i} x_{k^{\prime}}^{*} t_{i}^{\prime} y_{l^{\prime}} t_{i^{\prime}} \\
& +y_{k} t_{i} y_{l}^{*} t_{i} y_{k^{\prime}}^{*} t_{i}^{\prime} x_{l^{\prime}} t^{\prime}+y_{k} t_{i} y_{l}^{*} t_{i} y_{k^{\prime}}^{*} t_{i^{\prime}} y_{l^{\prime}} t_{i^{\prime}} \\
& +\frac{1}{L^{2}} \sum_{i=1}^{L} \sum_{i^{\prime}=1}^{L} x_{k} t_{i} y_{l}^{*} t_{i} x_{k^{\prime}}^{*} t_{i^{\prime}}+y_{k^{\prime}}^{*} t_{i} E\left[n_{l} t^{\prime}{ }^{\prime}\right] \\
& +x_{k} t_{i} y_{l}^{*} t_{i} x_{l^{\prime}} t_{i^{\prime}}+y_{l^{\prime}} t_{i^{\prime}} E\left[n_{k^{\prime}}^{*} t_{i^{\prime}}\right]+y_{k} t_{i} x_{l}^{*} t_{i} x_{k^{\prime}}^{*} t_{i^{\prime}}+y_{k^{\prime}}^{*} t_{i^{\prime}} E\left[n_{l^{\prime}} t_{i^{\prime}}\right] \\
& +y_{k} t_{i} x_{l}^{*} t_{i} x_{l} t_{i^{\prime}}+y_{l^{\prime}} t_{i} E\left[n_{k^{\prime}}^{*} t_{i}\right]+y_{k} t_{i} y_{l}^{*} t_{i} x_{k^{\prime}}^{*} t_{i^{\prime}}+y_{k^{\prime}}^{*} t_{i} E\left[n_{l^{\prime}} t^{\prime}\right]+y_{k} t_{i} y_{l}^{*} t_{i} x_{l^{\prime}} t_{i^{\prime}}+y_{l} t_{i} E\left[n_{k^{\prime}}^{*} t_{i^{\prime}}\right] \\
& +x_{k} t_{i}+y_{k} t_{i} x_{k^{\prime}}^{*} t_{i}^{\prime} y_{l^{\prime}} t_{i} E\left[n_{l}^{*} t_{i}\right]+x_{k} t_{i}+y_{k} t_{i} y_{k^{\prime}}^{*} t_{i}^{\prime} x_{l} t_{i} E\left[n_{l}^{*} t_{i}\right]+x_{k} t_{i}+y_{k} t_{i} y_{k^{\prime}}^{*} t_{i}^{\prime} y_{l} t_{i} E\left[n_{l}^{*} t_{i}\right] \\
& +x_{l}^{*} t_{i}+y_{l}^{*} t_{i} x_{k^{\prime}}^{*} t^{\prime} y_{l^{\prime}} t_{i}^{\prime} E\left[n_{k} t_{i}\right]+x_{l}^{*} t_{i}+y_{l}^{*} t_{i} y_{k^{\prime}}^{*} t_{i}^{\prime} x_{l^{\prime}} t_{i} E\left[n_{k} t_{i}\right]+x_{l}^{*} t_{i}+y_{l}^{*} t_{i} y_{k^{\prime}}^{*} t_{i}^{\prime} y_{l} t_{i} E\left[n_{k} t_{i}\right] \\
& +\frac{1}{L^{2}} \sum_{i=1}^{L} \sum_{i^{\prime}=1}^{L} x_{k} t_{i} y_{l}^{*} t_{i} E\left[n_{k^{\prime}}^{*} t_{i}^{\prime} n_{l^{\prime}} t_{i^{\prime}}\right] \\
& +y_{k^{\prime}}^{*} t_{i} x_{l^{\prime}} t_{i} E\left[n_{k} t_{i} n_{l}^{*} t_{i}\right]+y_{k^{\prime}}^{*} t_{i}^{\prime} y_{l^{\prime}} t_{i} E\left[n_{k} t_{i} n_{l}^{*} t_{i}\right] \\
& +y_{k} t_{i} y_{l}^{*} t_{i} E\left[n_{k^{\prime}}^{*} t_{i} n_{l^{\prime}} t_{i^{\prime}}\right]+x_{k} t_{i}+y_{k} t_{i} x_{k^{*}}^{*} t_{i^{\prime}}+y_{k^{\prime}}^{*} t_{i^{\prime}} \\
& \cdot E\left[n_{l}^{*} t_{i} n_{l^{\prime}} t_{i^{\prime}}\right]+x_{k} t_{i}+y_{k} t_{i} x_{l} t_{i^{\prime}}+y_{l} t_{i} E\left[n_{l}^{*} t_{i} n_{k^{\prime}}^{*} t_{i^{\prime}}\right] \\
& +x_{k^{\prime}}^{*} t_{i} y_{l} t_{i} E\left[n_{k} t_{i} n_{l}^{*} t_{i}\right] \\
& +\frac{1}{L^{2}} \sum_{i=1}^{L} \sum_{i^{\prime}=1}^{L} x_{k} t_{i}+y_{k} t_{i} E\left[n_{l}^{*} t_{i} n_{k^{\prime}}^{*} t_{i} n_{l^{\prime}} t_{i^{\prime}}\right] \\
& +x_{l}^{*} t_{i}+y_{l}^{*} t_{i} E\left[n_{k} t_{i} n_{k^{\prime}}^{*} t_{i}^{\prime} n_{l^{\prime}} t_{i^{\prime}}\right]+x_{k^{\prime}}^{*} t_{i^{\prime}}+y_{k^{\prime}}^{*} t_{i^{\prime}} E\left[n_{k} t_{i} n_{l}^{*} t_{i} n_{l^{\prime}} t_{i^{\prime}}\right] \\
& +x_{l^{\prime}} t_{i^{\prime}}+y_{l^{\prime}} t_{i} E\left[n_{k} t_{i} n_{l}^{*} t_{i} n_{k^{\prime}}^{*} t_{i^{\prime}}\right] \\
& +\frac{1}{L^{2}} \sum_{i=1}^{L} \sum_{i^{\prime}=1}^{L} E\left[n_{k} t_{i} n_{l}^{*} t_{i} n_{k^{\prime}}^{*} t_{i} n_{l} t_{i^{\prime}}\right] .
\end{aligned}
$$

From Appendix A and Appendix B, it is shown that the followings are identically zero:

$$
\begin{aligned}
x_{k} t_{i}+y_{k} t_{i} x_{l^{\prime}} t_{i^{\prime}}+y_{l^{\prime}} t_{i^{\prime}} E\left[n_{l}^{*} t_{i} n_{k^{\prime}}^{*} t_{i^{\prime}}\right] & =0, \\
x_{l}^{*} t_{i}+y_{l}^{*} t_{i} x_{k^{\prime}}^{*} t_{i^{\prime}}+y_{k^{\prime}}^{*} t_{i} E\left[n_{k} t_{i} n_{l^{\prime}} t_{i^{\prime}}\right] & =0, \\
x_{k} t_{i}+y_{k} t_{i} E\left[n_{l}^{*} t_{i} n_{k^{\prime}}^{*} t_{i^{\prime}} n_{l^{\prime}} t_{i^{\prime}}\right] & =0, \\
x_{l}^{*} t_{i}+y_{l}^{*} t_{i} E\left[n_{k} t_{i} n_{k^{\prime}}^{*} t_{i^{\prime}} n_{l^{\prime}} t_{i^{\prime}}\right] & =0, \\
x_{k^{\prime}}^{*} t_{i^{\prime}}+y_{k^{\prime}}^{*} t_{i^{\prime}} E\left[n_{k} t_{i} n_{l}^{*} t_{i} n_{l^{\prime}} t_{i^{\prime}}\right] & =0, \\
x_{l^{\prime}} t_{i^{\prime}}+y_{l^{\prime}} t_{i^{\prime}} E\left[n_{k} t_{i} n_{l}^{*} t_{i} n_{k^{\prime}}^{*} t_{i^{\prime}}\right] & =0 .
\end{aligned}
$$


Use (D.5) in (D.4), and (D.4) is simplified to

$$
\begin{aligned}
E\left[\delta \hat{R}_{k l} \delta \hat{R}_{k^{\prime} l^{\prime}}^{*}\right]= & \frac{1}{L^{2}} \sum_{i=1}^{L} \sum_{i^{\prime}=1}^{L} x_{k} t_{i} y_{l}^{*} t_{i} x_{k^{\prime}}^{*} t_{i^{\prime}} y_{l^{\prime}} t_{i^{\prime}}+x_{k} t_{i} y_{l}^{*} t_{i} y_{k^{\prime}}^{*} t_{i^{\prime}} x_{l^{\prime}} t_{i^{\prime}} \\
& +x_{k} t_{i} y_{l}^{*} t_{i} y_{k^{\prime}}^{*} t_{i^{\prime}} y_{l^{\prime}} t_{i^{\prime}}+y_{k} t_{i} x_{l}^{*} t_{i} x_{k^{\prime}}^{*} t_{i^{\prime}} y_{l^{\prime}} t_{i^{\prime}} \\
& +y_{k} t_{i} x_{l}^{*} t_{i} y_{k^{\prime}}^{*} t_{i^{\prime}} x_{l^{\prime}} t_{i^{\prime}}+y_{k} t_{i} x_{l}^{*} t_{i} y_{k^{\prime}}^{*} t_{i^{\prime}} y_{l^{\prime}} t_{i^{\prime}}+y_{k} \\
& t_{i} y_{l}^{*} t_{i} x_{k^{\prime}}^{*} t_{i^{\prime}} y_{l^{\prime}} t_{i^{\prime}}+y_{k} t_{i} y_{l}^{*} t_{i} y_{k^{\prime}}^{*} t_{i^{\prime}} x_{l^{\prime}} t_{i^{\prime}}+y_{k} t_{i} y_{l}^{*} t_{i} y_{k^{\prime}}^{*} t_{i^{\prime}} y_{l^{\prime}} t_{i^{\prime}} \\
& +\frac{1}{L^{2}} \sum_{i=1}^{L} \sum_{i^{\prime}=1}^{L} x_{k} t_{i} y_{l}^{*} t_{i} E\left[n_{k^{\prime}}^{*} t_{i^{\prime}} n_{l^{\prime}} t_{i^{\prime}}\right]+y_{k} t_{i^{\prime}} x_{l}^{*} t_{i} E\left[n_{k^{\prime}}^{*} t_{i^{\prime}} n_{l^{\prime}} t_{i^{\prime}}\right] \\
& +y_{k} t_{i} y_{l}^{*} t_{i} E\left[n_{k^{\prime}}^{*} t_{i^{\prime}} n_{l^{\prime}} t_{i^{\prime}}\right]+x_{k} t_{i}+y_{k} t_{i} x_{k^{\prime}}^{*} t_{i^{\prime}}+y_{k^{\prime}}^{*} t_{i^{\prime}} \\
& +E\left[n_{l}^{*} t_{i} n_{l^{\prime}} t_{i^{\prime}}\right]+y_{k^{\prime}}^{*} t_{i^{\prime}} y_{l^{\prime}} t^{\prime} E\left[n_{k} t_{i} n_{l}^{*} t_{i}\right] \\
& +x_{l}^{*} t_{i}+y_{l}^{*} t_{i} x_{l^{\prime}} t_{i^{\prime}}+y_{l^{\prime}} t_{i^{\prime}} E\left[n_{k} t_{i} n_{k^{\prime}}^{*} t_{i^{\prime}}\right]+y_{k^{\prime}}^{*} t_{i^{\prime}} x_{l^{\prime}} t_{i^{\prime}} E\left[n_{k} t_{i} n_{l}^{*} t_{i}\right] \\
& +x_{k^{\prime}}^{*} t_{i^{\prime}} y_{l^{\prime}} t_{i^{\prime}} E\left[n_{k} t_{i} n_{k}^{*} t_{i}\right]+E\left[n_{k} t_{i} n_{l}^{*} t_{i} n_{k^{\prime}}^{*} t_{i^{\prime}} n_{l^{\prime}} t_{i^{\prime}}\right]
\end{aligned}
$$

where the second moment and the fourth moment in (D.6) are derived in Appendix A and Appendix C, respectively.

(1) $k=l$ and $l=k^{\prime}$ and $k^{\prime}=l^{\prime}$ :

Finally, $E\left[(\delta \widehat{R})_{k l}(\delta \widehat{R})_{k^{\prime} l^{\prime}}^{*}\right]$ is given by

$$
\begin{aligned}
& x_{k} t_{i} y_{l}^{*} t_{i} x_{k^{\prime}}^{*} t_{i^{\prime}} y_{l^{\prime}} t_{i^{\prime}}+x_{k} t_{i} y_{l}^{*} t_{i} y_{k^{\prime}}^{*} t_{i^{\prime}} x_{l^{\prime}} t_{i^{\prime}}+x_{k} t_{i} y_{l}^{*} t_{i} y_{k^{\prime}}^{*} t_{i^{\prime}} \sum_{l^{\prime}} t_{i^{\prime}}+y_{k} t_{i} x_{l}^{*} t_{i} x_{k^{\prime}}^{*} t_{i^{\prime}} y_{l^{\prime}} t_{i^{\prime}}+y_{k} t_{i} x_{l}^{*} t_{i} y_{k^{\prime}}^{*} t_{i^{\prime}} x_{l^{\prime}} t_{i^{\prime}} t_{i} x_{l}^{*} t_{i} y_{k^{\prime}}^{*} t_{i^{\prime}} y_{l^{\prime}} t_{i^{\prime}}+y_{k} t_{i} y_{l}^{*} t_{i} x_{k^{\prime}}^{*} t_{i^{\prime}} y_{l^{\prime}} t_{i^{\prime}} \\
& +y_{k} t_{i} y_{l}^{*} t_{i} y_{k^{\prime}}^{*} t_{i^{\prime}} x_{l^{\prime}} t_{i^{\prime}}+y_{k} t_{i} y_{l}^{*} t_{i} y_{k^{\prime}}^{*} t_{i^{\prime}} y_{l^{\prime}} t_{i^{\prime}} \\
& +\frac{1}{L^{2}} \sigma^{2} \sum_{i=1}^{L} x_{k} t_{i} y_{l}^{*} t_{i}+y_{k} t_{i} x_{l}^{*} t_{i}+y_{k} t_{i} y_{l}^{*} t_{i} \\
& +x_{k} t_{i}+y_{k} t_{i} x_{k^{\prime}}^{*} t_{i}+y_{k^{\prime}}^{*} t_{i}+x_{l}^{*} t_{i}+y_{l}^{*} t_{i} x_{l^{\prime}} t_{i}+y_{l^{\prime}} t_{i} \\
& +x_{k^{\prime}}^{*} t_{i} y_{l^{\prime}} t_{i}+y_{k^{\prime}}^{*} t_{i} x_{l^{\prime}} t_{i}+y_{k^{\prime}}^{*} t_{i} y_{l^{\prime}} t_{i} \\
& +\frac{1}{L^{2}} \sigma^{2} \sum_{i=1}^{L} \sum_{i^{\prime}=1}^{L} x_{k} t_{i} y_{l}^{*} t_{i}+y_{k} t_{i} x_{l}^{*} t_{i}+y_{k} t_{i} y_{l}^{*} t_{i} \\
+ & x_{k^{\prime}}^{*} t_{i^{\prime}} y_{l^{\prime}} t_{i^{\prime}}+y_{k^{\prime}}^{*} t_{i^{\prime}} x_{l^{\prime}} t_{i^{\prime}}+y_{k^{\prime}}^{*} t_{i^{\prime}} y_{l^{\prime}} t_{i^{\prime}}+L^{2}-L \sigma^{4}
\end{aligned}
$$


(2) $k=l$ and $l=k^{\prime}$ and $k^{\prime} \neq l^{\prime}$ :

$$
\begin{aligned}
& x_{k} t_{i} y_{l}^{*} t_{i} x_{k^{\prime}}^{*} t_{i^{\prime}} y_{l} t_{i^{\prime}}+x_{k} t_{i} y_{l}^{*} t_{i} y_{k^{\prime}}^{*} t_{i}^{\prime} x_{l} t_{i}^{\prime} \\
& +x_{k} t_{i} y_{l}^{*} t_{i} y_{k^{\prime}}^{*} t_{i}^{\prime} y_{l} t_{i}+y_{k} t_{i} x_{l}^{*} t_{i} x_{k^{\prime}}^{*} t_{i} y_{l} t_{i}^{\prime} \\
& \frac{1}{L^{2}} \sum_{i=1}^{L} \sum_{i=1}^{L}+y_{k} t_{i} x_{l}^{*} t_{i} y_{k^{\prime}}^{*} t_{i} x_{l^{\prime}} t_{i}+y_{k} t_{i} x_{l}^{*} t_{i} y_{k^{\prime}}^{*} t_{i} y_{l^{\prime}} t^{\prime} \\
& +y_{k} t_{i} y_{l}^{*} t_{i} x_{k^{\prime}}^{*} t_{i^{\prime}} y_{l^{\prime}} t_{i^{\prime}}+y_{k} t_{i} y_{l}^{*} t_{i} y_{k^{\prime}}^{*} t_{i^{\prime}} x_{l^{\prime}} t_{i^{\prime}} \\
& +y_{k} t_{i} y_{l}^{*} t_{i} y_{k^{\prime}}^{*} t_{i^{\prime}} y_{l^{\prime}} t_{i} \\
& +\sigma^{2} \frac{1}{L^{2}} \sum_{i=1}^{L} x_{l}^{*} t_{i}+y_{l}^{*} t_{i} x_{l} t_{i}+y_{l} t_{i} \\
& +x_{k^{\prime}}^{*} t_{i} y_{l} t_{i}+y_{k^{\prime}}^{*} t_{i} x_{l} t_{i}+y_{k^{\prime}}^{*} t_{i} y_{l} t_{i} \\
& +\sigma^{2} \frac{1}{L^{2}} \sum_{i=1}^{L} \sum_{i^{\prime}=1}^{L} x_{k^{\prime}}^{*} t_{i^{\prime}} y_{l^{\prime}} t_{i^{\prime}}+y_{k^{\prime}}^{*} t_{i^{\prime}} x_{l} t_{i^{\prime}}+y_{k^{\prime}}^{*} t_{i^{\prime}} y_{l^{\prime}} t_{i^{\prime}} .
\end{aligned}
$$

(3) $k=l$ and $l=l^{\prime}$ and $l^{\prime} \neq k^{\prime}$ :

$$
\begin{aligned}
& x_{k} t_{i} y_{l}^{*} t_{i} x_{k^{\prime}}^{*} t_{i^{\prime}} y_{l^{\prime}} t_{i^{\prime}}+x_{k} t_{i} y_{l}^{*} t_{i} y_{k^{\prime}}^{*} t_{i^{\prime}} x_{l^{\prime}} t_{i^{\prime}} \\
& +x_{k} t_{i} y_{l}^{*} t_{i} y_{k^{\prime}}^{*} t_{i}^{\prime} y_{l} t_{i^{\prime}}+y_{k} t_{i} x_{l}^{*} t_{i} x_{k^{\prime}}^{*} t_{i}^{\prime} y_{l} t_{i^{\prime}} \\
& \frac{1}{L^{2}} \sum_{i=1}^{L} \sum_{i^{\prime}=1}^{L}+y_{k} t_{i} x_{l}^{*} t_{i} y_{k^{\prime}}^{*} t_{i} x_{l^{\prime}} t_{i^{\prime}}+y_{k} t_{i} x_{l}^{*} t_{i} y_{k^{\prime}}^{*} t_{i^{\prime}} y_{l^{\prime}} t_{i^{\prime}} \\
& +y_{k} t_{i} y_{l}^{*} t_{i} x_{k^{\prime}}^{*} t_{i^{\prime}} y_{l^{\prime}} t_{i^{\prime}}+y_{k} t_{i} y_{l}^{*} t_{i} y_{k^{\prime}}^{*} t_{i}^{\prime} x_{l^{\prime}} t_{i^{\prime}} \\
& +y_{k} t_{i} y_{l}^{*} t_{i} y_{k^{\prime}}^{*} t_{i^{\prime}} y_{l^{\prime}} t_{i^{\prime}} \\
& +\sigma^{2} \frac{1}{L^{2}} \sum_{i=1}^{L} x_{k} t_{i}+y_{k} t_{i} x_{k^{\prime}}^{*} t_{i}+y_{k^{\prime}}^{*} t_{i} \\
& +x_{k^{\prime}}^{*} t_{i} y_{l^{\prime}} t_{i}+y_{k^{\prime}}^{*} t_{i} x_{l^{\prime}} t_{i}+y_{k^{\prime}}^{*} t_{i} y_{l^{\prime}} t_{i} \\
& +\sigma^{2} \frac{1}{L^{2}} \sum_{i^{\prime}=1}^{L} \sum_{i=1}^{L} x_{k^{\prime}}^{*} t_{i^{\prime}} y_{l} t_{i^{\prime}}+y_{k^{\prime}}^{*} t_{i} x_{l} t_{i^{\prime}}+y_{k^{\prime}}^{*} t_{i} y_{l^{\prime}} t_{i^{\prime}} .
\end{aligned}
$$

(4) $k=k^{\prime}$ and $k^{\prime}=l^{\prime}$ and $l^{\prime} \neq l$ :

$$
\begin{aligned}
& x_{k} t_{i} y_{l}^{*} t_{i} x_{k^{\prime}}^{*} t_{i}^{\prime} y_{l^{\prime}} t_{i}+x_{k} t_{i} y_{l}^{*} t_{i} y_{k^{\prime}}^{*} t_{i} x_{l^{\prime}} t_{i} \\
& +x_{k} t_{i} y_{l}^{*} t_{i} y_{k^{\prime}}^{*} t_{i}^{\prime} y_{l} t_{i}+y_{k} t_{i} x_{l}^{*} t_{i} x_{k^{\prime}}^{*} t_{i} y_{l} t_{i} \\
& \frac{1}{L^{2}} \sum_{i=1}^{L} \sum_{i^{\prime}=1}^{L}+y_{k} t_{i} x_{l}^{*} t_{i} y_{k^{\prime}}^{*} t_{i} x_{l^{\prime}} t_{i}+y_{k} t_{i} x_{l}^{*} t_{i} y_{k^{\prime}}^{*} t_{i} y_{l^{\prime}} t_{i} \\
& +y_{k} t_{i} y_{l}^{*} t_{i} x_{k^{\prime}}^{*} t_{i} y_{l} t_{i}+y_{k} t_{i} y_{l}^{*} t_{i} y_{k^{\prime}}^{*} t_{i} x_{l} t_{i} \\
& +y_{k} t_{i} y_{l}^{*} t_{i} y_{k^{\prime}}^{*} t_{i} y_{l^{\prime}} t_{i^{\prime}} \\
& +\sigma^{2} \frac{1}{L^{2}} \sum_{i=1}^{L} x_{k} t_{i} y_{l}^{*} t_{i}+y_{k} t_{i} x_{l}^{*} t_{i} \\
& +y_{k} t_{i} y_{l}^{*} t_{i}+x_{l}^{*} t_{i}+y_{l}^{*} t_{i} x_{l} t_{i}+y_{l} t_{i} \\
& +\sigma^{2} \frac{1}{L^{2}} \sum_{i=1}^{L} \sum_{i=1}^{L} x_{k} t_{i} y_{l}^{*} t_{i}+y_{k} t_{i} x_{l}^{*} t_{i}+y_{k} t_{i} y_{l}^{*} t_{i} .
\end{aligned}
$$

(5) $l=k^{\prime}$ and $k^{\prime}=l^{\prime}$ and $l^{\prime} \neq k$ :

$$
\begin{aligned}
& k t_{i} y_{l}^{*} t_{i} x_{k^{\prime}}^{*} t_{i} y_{l^{\prime}} t_{i}+x_{k} t_{i} y_{l}^{*} t_{i} y_{k^{\prime}}^{*} t_{i} x_{l^{\prime}} t_{i^{\prime}} \\
& +x_{k} t_{i} y_{l}^{*} t_{i} y_{k^{\prime}}^{*} t_{i}^{\prime} y_{l} t_{i}^{\prime}+y_{k} t_{i} x_{l}^{*} t_{i} x_{k^{\prime}}^{*} t_{i}^{\prime} y_{l} t_{i} \\
& \frac{1}{L^{2}} \sum_{i=1}^{L} \sum_{i^{\prime}=1}^{L}+y_{k} t_{i} x_{l}^{*} t_{i} y_{k^{\prime}}^{*} t_{i} x_{l} t_{i}+y_{k} t_{i} x_{l}^{*} t_{i} y_{k^{\prime}}^{*} t_{i} y_{l^{\prime}} t_{i^{\prime}} \\
& +y_{k} t_{i} y_{l}^{*} t_{i} x_{k^{\prime}}^{*} t_{i^{\prime}} y_{l^{\prime}} t_{i^{\prime}}+y_{k} t_{i} y_{l}^{*} t_{i} y_{k^{\prime}}^{*} t_{i}^{\prime} x_{l^{\prime}} t_{i^{\prime}} \\
& +y_{k} t_{i} y_{l}^{*} t_{i} y_{k^{\prime}}^{*} t_{i}^{\prime} y_{l^{\prime}} t_{i^{\prime}} \\
& +\sigma^{2} \frac{1}{L^{2}} \sum_{i=1}^{L} x_{k} t_{i} y_{l}^{*} t_{i}+y_{k} t_{i} x_{l}^{*} t_{i} \\
& +y_{k} t_{i} y_{l}^{*} t_{i}+x_{k} t_{i}+y_{k} t_{i} x_{k^{\prime}}^{*} t_{i}+y_{k^{\prime}}^{*} t_{i} \\
& +\sigma^{2} \frac{1}{L^{2}} \sum_{i=1}^{L} \sum_{i=1}^{L} x_{k} t_{i} y_{l}^{*} t_{i}+y_{k} t_{i} x_{l}^{*} t_{i}+y_{k} t_{i} y_{l}^{*} t_{i} .
\end{aligned}
$$


(6) $k=l$ and $l \neq k^{\prime}$ and $k^{\prime}=l^{\prime}$ :

$$
\begin{aligned}
& x_{k} t_{i} y_{l}^{*} t_{i} x_{k^{\prime}}^{*} t_{i}^{\prime} y_{l} t_{i}^{\prime}+x_{k} t_{i} y_{l}^{*} t_{i} y_{k^{\prime}}^{*} t_{i}^{\prime} x_{l^{\prime}} t_{i}^{\prime} \\
& +x_{k} t_{i} y_{l}^{*} t_{i} y_{k^{\prime}}^{*} t_{i^{\prime}} y_{l^{\prime}} t_{i^{\prime}}+y_{k} t_{i} x_{l}^{*} t_{i} x_{k^{\prime}}^{*} t_{i^{\prime}} y_{l^{\prime}} t_{i^{\prime}} \\
& \frac{1}{L^{2}} \sum_{i=1}^{L} \sum_{i^{\prime}=1}^{L}+y_{k} t_{i} x_{l}^{*} t_{i} y_{k^{\prime}}^{*} t_{i} x_{l^{\prime}} t^{\prime}+y_{k} t_{i} x_{l}^{*} t_{i} y_{k^{\prime}}^{*} t_{i}^{\prime} y_{l^{\prime}} t^{\prime} \\
& +y_{k} t_{i} y_{l}^{*} t_{i} x_{k^{\prime}}^{*} t_{i} y_{l} t_{i^{\prime}}+y_{k} t_{i} y_{l}^{*} t_{i} y_{k^{\prime}}^{*} t_{i} x_{l^{\prime}} t_{i^{\prime}} \\
& +y_{k} t_{i} y_{l}^{*} t_{i} y_{k^{\prime}}^{*} t_{i}^{\prime} y_{l^{\prime}} t_{i}^{\prime} \\
& +\frac{1}{L^{2}} \sigma^{2} \sum_{i=1}^{L} x_{k} t_{i} y_{l}^{*} t_{i}+y_{k} t_{i} x_{l}^{*} t_{i}+y_{k} t_{i} y_{l}^{*} t_{i} \\
& +x_{k^{\prime}}^{*} t_{i} y_{l} t_{i}+y_{k^{\prime}}^{*} t_{i} x_{l^{\prime}} t_{i}+y_{k^{\prime}}^{*} t_{i} y_{l^{\prime}} t_{i}+L \sigma^{4} \text {. }
\end{aligned}
$$

(7) $k=k^{\prime}$ and $k^{\prime} \neq l$ and $l=l^{\prime}$ :

$$
\begin{gathered}
x_{k} t_{i} y_{l}^{*} t_{i} x_{k^{\prime}}^{*} t_{i}^{\prime} y_{l^{\prime}} t_{i^{\prime}}+x_{k} t_{i} y_{l}^{*} t_{i} y_{k^{\prime}}^{*} t_{i} x_{l^{\prime}} t_{i^{\prime}} \\
+x_{k} t_{i} y_{l}^{*} t_{i} y_{k^{\prime}}^{*} t_{i^{\prime}} y_{l^{\prime}} t_{i^{\prime}}+y_{k} t_{i} x_{l}^{*} t_{i} x_{k^{\prime}}^{*} t_{i^{\prime}} y_{l^{\prime}} t_{i^{\prime}} \\
\frac{1}{L^{2} \sum_{i=1}^{L} \sum_{i^{\prime}=1}^{L}}+y_{k} t_{i} x_{l}^{*} t_{i} y_{k^{\prime}}^{*} t_{i^{\prime}} x_{l^{\prime}} t_{i^{\prime}}+y_{k} t_{i} x_{l}^{*} t_{i} y_{k^{\prime}}^{*} t_{i^{\prime}} y_{l^{\prime}} t_{i^{\prime}} \\
+y_{k} t_{i} y_{l}^{*} t_{i} x_{k^{\prime}}^{*} t_{t^{\prime}} y_{l^{\prime}} t_{i^{\prime}}+y_{k} t_{i} y_{l}^{*} t_{i} y_{k^{\prime}}^{*} t_{i^{\prime}} x_{l^{\prime}} t_{i^{\prime}} \\
+y_{k} t_{i} y_{l}^{*} t_{i} y_{k^{\prime}}^{*} t^{\prime} y_{l^{\prime}} t_{i^{\prime}} \\
+\frac{1}{L^{2}} \sigma^{2} \sum_{i=1}^{L} x_{k} t_{i}+y_{k} t_{i} x_{k^{\prime}}^{*} t_{i}+y_{k^{\prime}}^{*} t_{i} \\
+x_{l}^{*} t_{i}+y_{l}^{*} t_{i} x_{l^{\prime}} t_{i}+y_{l^{\prime}} t_{i}+L \sigma^{4} .
\end{gathered}
$$

(8) $k=k^{\prime}$ and $k^{\prime} \neq l$ and $l \neq l^{\prime}$ and $l^{\prime}$ :

$$
\begin{aligned}
& x_{k} t_{i} y_{l}^{*} t_{i} x_{k^{\prime}}^{*} t_{i}^{\prime} y_{l^{\prime}} t_{i^{\prime}}+x_{k} t_{i} y_{l}^{*} t_{i} y_{k^{\prime}}^{*} t_{i}^{\prime} x_{l^{\prime}} t_{i} \\
& +x_{k} t_{i} y_{l}^{*} t_{i} y_{k^{\prime}}^{*} t_{i} y_{l} t_{i}+y_{k} t_{i} x_{l}^{*} t_{i} x_{k^{\prime}}^{*} t_{i}^{\prime} y_{l} t_{i} \\
& \frac{1}{L^{2}} \sum_{i=1}^{L} \sum_{i=1}^{L}+y_{k} t_{i} x_{l}^{*} t_{i} y_{k^{\prime}}^{*} t_{i} x_{l^{\prime}} t_{i}+y_{k} t_{i} x_{l}^{*} t_{i} y_{k^{\prime}}^{*} t^{\prime} y_{l} t_{i} \\
& +y_{k} t_{i} y_{l}^{*} t_{i} x_{k^{\prime}}^{*} t_{i}^{\prime} y_{l} t_{i}+y_{k} t_{i} y_{l}^{*} t_{i} y_{k}^{*} t_{i}^{\prime} x_{l} t_{i} \\
& +y_{k} t_{i} y_{l}^{*} t_{i} y_{k^{\prime}}^{*} t_{i^{\prime}} y_{l^{\prime}} t_{i^{\prime}} \\
& +\sigma^{2} \frac{1}{L^{2}} \sum_{i=1}^{L} x_{l}^{*} t_{i}+y_{l}^{*} t_{i} x_{l} t_{i}+y_{l} t_{i} .
\end{aligned}
$$

(9) $k^{\prime}=l^{\prime}$ and $l^{\prime} \neq k$ and $k \neq l$ and $l \neq k^{\prime}$ :

$$
\begin{aligned}
& x_{k} t_{i} y_{l}^{*} t_{i} x_{k^{\prime}}^{*} t_{i}^{\prime} y_{l} t_{i}^{\prime}+x_{k} t_{i} y_{l}^{*} t_{i} y_{k^{\prime}}^{*} t_{i}^{\prime} x_{l^{\prime}} t_{i^{\prime}} \\
& +x_{k} t_{i} y_{l}^{*} t_{i} y_{k^{\prime}}^{*} t_{i^{\prime}} y_{l^{\prime}} t_{i^{\prime}}+y_{k} t_{i} x_{l}^{*} t_{i} x_{k^{\prime}}^{*} t_{i^{\prime}} y_{l^{\prime}} t_{i^{\prime}} \\
& \frac{1}{L^{2}} \sum_{i=1}^{L} \sum_{i=1}^{L}+y_{k} t_{i} x_{l}^{*} t_{i} y_{k^{\prime}}^{*} t_{i} x_{l} t_{i^{\prime}}+y_{k} t_{i} x_{l}^{*} t_{i} y_{k^{\prime}}^{*} t_{i} y_{l} t^{\prime} \\
& +y_{k} t_{i} y_{l}^{*} t_{i} x_{k^{\prime}}^{*} t_{i}^{\prime} y_{l^{\prime}} t_{i}^{\prime}+y_{k} t_{i} y_{l}^{*} t_{i} y_{k^{\prime}}^{*} t_{i}^{\prime} x_{l^{\prime}} t_{i^{\prime}} \\
& +y_{k} t_{i} y_{l}^{*} t_{i} y_{k^{\prime}}^{*} t_{i^{\prime}} y_{l^{\prime}} t_{i^{\prime}} \\
& +\sigma^{2} \frac{1}{L^{2}} \sum_{i=1}^{L} x_{k} t_{i} y_{l}^{*} t_{i}+y_{k} t_{i} x_{l}^{*} t_{i}+y_{k} t_{i} y_{l}^{*} t_{i} \\
& +\frac{1}{L^{2}} \sigma^{2} \sum_{i=1}^{L} \sum_{i^{\prime}=1}^{L} x_{k} t_{i} y_{l}^{*} t_{i}+y_{k} t_{i} x_{l}^{*} t_{i}+y_{k} t_{i} y_{l}^{*} t_{i} .
\end{aligned}
$$

(10) $l=l^{\prime}$ and $l^{\prime} \neq k$ and $k \neq k^{\prime}$ and $k^{\prime} \neq l$ :

$$
\begin{gathered}
x_{k} t_{i} y_{l}^{*} t_{i} x_{k^{\prime}}^{*} t_{i^{\prime}} y_{l^{\prime}} t_{i^{\prime}}+x_{k} t_{i} y_{l}^{*} t_{i} y_{k^{\prime}}^{*} t_{i} x_{l^{\prime}} t^{\prime} \\
+x_{k} t_{i} y_{l}^{*} t_{i} y_{k^{\prime}}^{*} t^{\prime} y_{l^{\prime}} t_{i^{\prime}}+y_{k} t_{i} x_{l}^{*} t_{i} x_{k^{\prime}}^{*} t_{i^{\prime}} y_{l^{\prime}} t_{i^{\prime}} \sum_{i=1}^{L} \sum_{i^{\prime}=1}^{L}+y_{k} t_{i} x_{l}^{*} t_{i} y_{k^{\prime}}^{*} t_{i^{\prime}} x_{l^{\prime}} t_{i^{\prime}}+y_{k} t_{i} x_{l}^{*} t_{i} y_{k^{\prime}}^{*} t_{i^{\prime}} y_{l^{\prime}} t_{i^{\prime}} \\
+y_{k} t_{i} y_{l}^{*} t_{i} x_{k^{\prime}}^{*} t_{i^{\prime}} y_{l^{\prime}} t_{i^{\prime}}+y_{k} t_{i} y_{l}^{*} t_{i} y_{k^{\prime}}^{*} t_{i^{\prime}} x_{l^{\prime}} t_{i^{\prime}} \\
+y_{k} t_{i} y_{l}^{*} t_{i} y_{k^{\prime}}^{*} t_{i^{\prime}} y_{l^{\prime}} t_{i^{\prime}} \\
+\sigma^{2} \frac{1}{L^{2}} \sum_{i=1}^{L} x_{k} t_{i}+y_{k} t_{i} x_{k^{\prime}}^{*} t_{i}+y_{k^{\prime}}^{*} t_{i^{\prime}} .
\end{gathered}
$$

(11) $k=l$ and $l \neq k^{\prime}$ and $k^{\prime} \neq l^{\prime}$ and $l^{\prime} \neq k$ :

$x_{k} t_{i} y_{l}^{*} t_{i} x_{k^{\prime}}^{*} t_{i}^{\prime} y_{l} t_{i^{\prime}}+x_{k} t_{i} y_{l}^{*} t_{i} y_{k^{\prime}}^{*} t_{i} x_{l^{\prime}} t^{\prime}$

$+x_{k} t_{i} y_{l}^{*} t_{i} y_{k^{\prime}}^{*} t_{i^{\prime}} y_{l^{\prime}} t_{i^{\prime}}+y_{k} t_{i} x_{l}^{*} t_{i} x_{k^{\prime}}^{*} t_{i^{\prime}} y_{l^{\prime}} t_{i}$

$\frac{1}{L^{2}} \sum_{i=1}^{L} \sum_{i^{\prime}=1}^{L}+y_{k} t_{i} x_{l}^{*} t_{i} y_{k^{\prime}}^{*} t_{i} x_{l^{\prime}} t_{i^{\prime}}+y_{k} t_{i} x_{l}^{*} t_{i} y_{k^{\prime}}^{*} t^{\prime} y_{l} t_{i^{\prime}}$

$+y_{k} t_{i} y_{l}^{*} t_{i} x_{k^{\prime}}^{*} t_{i^{\prime}} y_{l^{\prime}} t_{i^{\prime}}+y_{k} t_{i} y_{l}^{*} t_{i} y_{k^{\prime}}^{*} t_{i}^{\prime} x_{l} t_{i^{\prime}}$

$+y_{k} t_{i} y_{l}^{*} t_{i} y_{k^{\prime}}^{*} t_{i}^{\prime} y_{l^{\prime}} t_{i}^{\prime}$

$+\sigma^{2} \frac{1}{L^{2}} \sum_{i=1}^{L} x_{k^{\prime}}^{*} t_{i} y_{l} t_{i}+y_{k^{\prime}}^{*} t_{i} x_{l} t_{i}+y_{k^{\prime}}^{*} t_{i} y_{l} t_{i}$

$+\frac{1}{L^{2}} \sigma^{2} \sum_{i=1}^{L} \sum_{i^{\prime}=1}^{L} x_{k^{\prime}}^{*} t_{i^{\prime}} y_{l^{\prime}} t_{i^{\prime}}+y_{k^{\prime}}^{*} t_{i} x_{l^{\prime}} t_{i^{\prime}}+y_{k^{\prime}}^{*} t_{i^{\prime}} y_{l^{\prime}} t_{i^{\prime}}$. 
(12) Otherwise,

$$
\begin{gathered}
x_{k} t_{i} y_{l}^{*} t_{i} x_{k^{\prime}}^{*} t_{i^{\prime}} y_{l^{\prime}} t_{i^{\prime}}+x_{k} t_{i} y_{l}^{*} t_{i} y_{k^{\prime}}^{*} t_{i^{\prime}} x_{l^{\prime}} t_{i^{\prime}} \\
+x_{k} t_{i} y_{l}^{*} t_{i} y_{k^{\prime}}^{*} t_{i^{\prime}} y_{l^{\prime}} t_{i^{\prime}}+y_{k} t_{i} x_{l}^{*} t_{i} x_{k^{\prime}}^{*} t_{i^{\prime}} y_{l^{\prime}} t_{i^{\prime}}^{L} \sum_{i=1}^{L} \sum_{i^{\prime}=1}^{L}+y_{k} t_{i} x_{l}^{*} t_{i} y_{k^{\prime}}^{*} t_{i^{\prime}} x_{l^{\prime}} t_{i^{\prime}}+y_{k} t_{i} x_{l}^{*} t_{i} y_{k^{\prime}}^{*} t_{i^{\prime}} y_{l^{\prime}} t_{i^{\prime}} \cdot \\
+y_{k} t_{i} y_{l}^{*} t_{i} x_{k^{\prime}}^{*} t_{i^{\prime}} y_{l^{\prime}} t_{i^{\prime}}+y_{k} t_{i} y_{l}^{*} t_{i} y_{k^{\prime}}^{*} t_{i^{\prime}} x_{l^{\prime}} t_{i^{\prime}} \\
+y_{k} t_{i} y_{l}^{*} t_{i} y_{k^{\prime}}^{*} t_{i^{\prime}} y_{l^{\prime}} t_{i^{\prime}}
\end{gathered}
$$

\section{Data Availability}

The data used to support the findings of this study are available from the corresponding author upon request.

\section{Conflicts of Interest}

The authors declare that there are no conflicts of interest regarding the publication of this paper.

\section{Authors' Contributions}

Eun-kyung Lee made a Matlab simulation. Joon-Ho Lee wrote an initial draft. Joon-Ho Lee and Eun-kyung Lee corrected the manuscript. Joon-Ho Lee originally derived the mathematical formulation of the proposed scheme.

\section{Acknowledgments}

This research was supported by Basic Science Research Program through the National Research Foundation of Korea (NRF) funded by the Ministry of Education (2018R1D1A1B07048294) and the National Research Foundation of Korea (NRF) grant funded by the Korea government (MSIT) (No. 2020R1A2C1009435).

\section{References}

[1] J.-H. Lee, S.-W. Cho, and H. S. Kim, "Newton-type method in spectrum estimaion-based AOA estimation," IEICE Electronics Express, vol. 9, no. 12, pp. 1036-1043, 2012.

[2] H. Krim and M. Viberg, "Two decades of array signal processing research-the parametric approach," IEEE Signal Processing Magazine, vol. 13, no. 7, pp. 67-94, 1996.

[3] J. Capon, "High-resolution frequency-wavenumber spectrum analysis," Proceedings of the IEEE, vol. 57, no. 8, pp. 1408-1418, 1969.

[4] S. Lang and J. McClellan, "Frequency estimation with maximum entropy spectral estimators," IEEE Transactions on Acoustics, Speech, and Signal Processing, vol. 28, no. 6, pp. 716-724, 1980.

[5] R. O. Schmidt, "Multiple emitter location and signal parameter estimation," in Proceedings of RADC Spectral Estimation Workshop, pp. 243-258, Rome, Italy, October 1979.

[6] R. Roy, A. Paulraj, and T. Kailath, "Esprit-a subspace rotation approach to estimation of parameters of cisoids in noise," IEEE Transactions on Acoustics, Speech, and Signal Processing, vol. 34, no. 5, pp. 1340-1342, 1986.
[7] I. Ziskind and M. Wax, "Maximum likelihood localization of multiple sources by alternating projection," IEEE Transactions on Acoustics, Speech, and Signal Processing, vol. 36, no. 10, pp. 1553-1560, 1988.

[8] T. C. Yang, "Deconvolved conventional beamforming for a horizontal line array," IEEE Journal of Oceanic Engineering, vol. 43, no. 1, pp. 160-172, 2018.

[9] Z. Szalay and L. Nagy, “Target modeling, antenna array design and conventional beamforming algorithms for radar target DOA estimation," in Proceedings of 2015 17th International Conference on Transparent Optical Networks (ICTON), Budapest, Hungary, July 2015.

[10] T. C. Yang, "On conventional beamforming and deconvolution," in Proceedings of OCEANS 2016, Shanghai, China, April 2016.

[11] J. Benesty, J. Chen, and Y. Huang, "Conventional beamforming techniques," Springer Topics in Signal Processing, Springer, Berlin, Germany, 2008.

[12] V. Krishnaveni, T. Kesavamurthy, and B. Apama, "Beamforming for direction-of-arrival (DOA) estimation-a survey," International Journal of Computer Applications, vol. 61, no. 11, 2013.

[13] Z. Xu, G. White, and Y. Zakharov, "Optimisation of beam pattern of high-altitude platform antenna using conventional beamforming," IEE Proceedings-Communications, vol. 153, no. 6, pp. 865-870, 2006.

[14] D. Sun, C. Ma, J. Mei, W. Sh, and L. Gao, "The deconvolved conventional beamforming for non-uniform line arrays," in Proceedings of 2018 Oceans-MTS/IEEE Kobe Techno-Oceans (OTO), Kobe, Japan, May 2018.

[15] G. Jiang, C. Sun, and X. Liu, "Diagonal denoising for conventional beamforming via sparsity optimization," IEEE Access, vol. 8, p. 11416, Article ID 11425, 2020.

[16] H. Jin, C. Jianwen, and L. Zhong, "Improve spectral resolution of subspace-based DOA estimation algorithms using conventional beamforming by noise subspace projection," in Proceedings of IEEE Antennas and Propagation Society Symposium, Monerey, CA, USA, 2004.

[17] R. Otnes, "Projection-based tapering for conventional beamforming on nonlinear sonar arrays," in Proceedings of Oceans 2010, Seattle, WA, USA, September 2010.

[18] D. Christ, "Cross coherence and joint pdf of the bartlett and capon power spectral estimates," in Proceedings of IEEE International Conference on Acoustics, Speech and Signal Processing-ICASSP '07, Honolulu, HI, USA, April 2007.

[19] D. Christ, "Statistical analysis of capon-bartlett 2-D cross spectrum," in Proceedings of IEEE International Conference on Acoustics, Speech and Signal Processing - ICASSP 2010, Dallas, TX, USA, March 2010.

[20] S. Haykin, T. V. Ho, J. Litva et al., Radar Array Processing, Springer, Berlin, Germany, 1993.

[21] S. De Graaf and D. Johnson, "Capability of array processing algorithms to estimate source bearings," IEEE Transactions on Acoustics, Speech, and Signal Processing, vol. 33, no. 6, pp. 1368-1379, 1985. 